\title{
A Free Energy Model for Hysteresis in Ferroelectric Materials
}

\author{
Ralph C. Smith \\ Department of Mathematics \\ Center for Research in Scientific Computation \\ North Carolina State University \\ Raleigh, NC 27695 \\ rsmith@eos.ncsu.edu \\ Zoubeida Ounaies \\ Department of Mechanical Engineering \\ Virginia Commonwealth University \\ Richmond VA 23284-3015 \\ zounaies@vcu.edu
}

\author{
Stefan Seelecke \\ Mechanical and Aerospace Engineering \\ Center for Research in Scientific Computation \\ North Carolina State University \\ Raleigh, NC 27695 \\ stefan_seelecke@ncsu.edu
}

\begin{abstract}
This paper provides a theory for quantifying the hysteresis and constitutive nonlinearities inherent to piezoceramic compounds through a combination of free energy analysis and stochastic homogenization techniques. In the first step of the model development, Helmholtz and Gibbs free energy relations are constructed at the lattice or domain level to quantify the relation between the field and polarization in homogeneous, single crystal compounds which exhibit uniform effective fields. The effects of material nonhomogeneities, polycrystallinity, and variable effective fields are subsequently incorporated through the assumption that certain physical parameters, including the local coercive and effective fields, are randomly distributed and hence manifestations of stochastic density functions associated with the material. Stochastic homogenization in this manner provides low-order macroscopic models with effective parameters that can be correlated with physical properties of the data. This facilitates the identification of parameters for model construction, model updating to accommodate changing operating conditions, and control design utilizing model-based inverse compensators. Attributes of the model, including the guaranteed closure of biased minor loops in quasistatic drive regimes, are illustrated through examples.
\end{abstract}




\section{Introduction}

The capability of piezoelectric materials to both actuate and sense derives from the noncentrosymmetric nature of the compounds. At very low input field levels, changes in the ionic structure produce reversible changes in the polarization whereas dipole switching at higher field inputs yields irreversible increments in the polarization. This generates strains in the material and provides it with actuator capabilities. Alternatively, applied stresses also alter the ionic configuration which generates the voltages measured in piezoelectric sensors. The two mechanisms are respectively termed the converse and direct piezoelectric effects.

The coupled converse and direct electromechanical effects are highly sensitive and repeatable which makes PZT transducers the present choice for applications such as nanopositioning and sensing. For example, the PZT positioning elements in an atomic force microscope (AFM) can be used to achieve angstrom-level displacements while PZT sensors are presently being investigated for use in multistage nanopositioners [7]. However, the polar mechanisms which provide piezoelectric materials with the dual converse and direct effects, and extreme electromechanical sensitivity, also produce varying degrees of hysteresis and constitutive nonlinearities as illustrated in Figure 1 for PZT5A. Hysteresis is an inherent property of noncentrosymmetric compounds at all drive levels and is due to the irreversible changes which accompany dipole switching; furthermore, saturation at the domain level and material nonhomogeneities contribute nonlinear effects. Both the hysteresis and constitutive nonlinearities must be accommodated for high performance applications utilizing PZT actuators and sensors.

For a broad range of applications, feedback laws can be employed to mitigate the deleterious effects of hysteresis and constitutive nonlinearities. This has led to the successful use of piezoelectric transducers in applications ranging from hybrid motor design to structural acoustic control (e.g., see $[4,9,14,38])$. In other regimes, however, noise to signal ratios and fundamental control issues limit the degree to which feedback design can solely be employed to linearize the response of piezoelectric actuators. For example, the positioning elements in atomic force microscopes and nanopositioners are comprised of stacked or cylindrical PZT actuators. At low drive frequencies, high gain feedback laws are presently employed to attenuate hysteresis and nonlinearities thus leading to the phenomenal

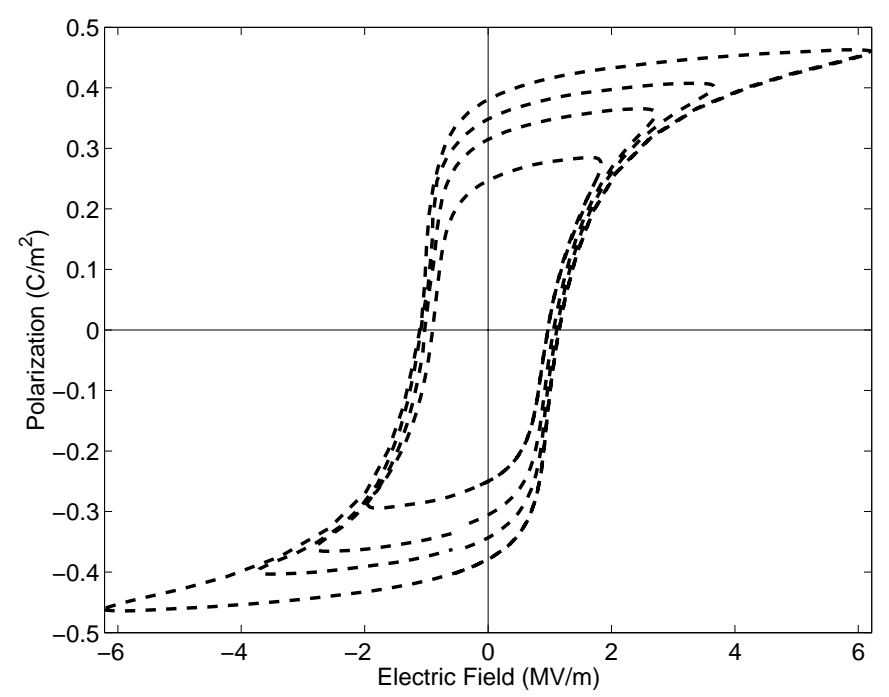

Figure 1. Hysteresis measured at $200 \mathrm{mHz}$ in PZT5A for peak inputs of $600 \mathrm{~V}, 800 \mathrm{~V}, 1000 \mathrm{~V}$ and $1600 \mathrm{~V}$. 
success of the devices. However, at the higher frequencies required for large scale product diagnostics or real-time monitoring of biological processes, the efficacy of feedback laws is diminished by inherent thermal and measurement noise. Robust control design can be used to extend the frequency ranges of operation [27], but the loop shaping and gains required to attenuate hysteresis have the negative effect of accentuating high frequency noise. One technique for circumventing these limitations is to develop feedforward or feedback loops which utilize highly accurate and efficient model-based inverse compensators. Models designed for this use must satisfy at least three properties: (i) they must accommodate transient dynamics, (ii) they must guarantee closure of biased minor loops, and (iii) they must be sufficiently efficient to permit real-time control implementation. In this paper, we develop a macroscopic polarization model through the combination of free energy principles at the lattice level and stochastic homogenization techniques which guarantees properties (i) and (ii). Furthermore, initial investigations attest to its potential for real-time control implementation (iii).

To provide a context for the approach, we first summarize techniques that have recently been developed for quantifying the hysteretic field-polarization relation in piezoelectric materials. These techniques can be roughly categorized as employing energy principles, phenomenological principles, or a combination of the two, to construct microscopic, mesoscopic (domain or lattice level), or macroscopic models.

Microscopic and mesoscopic models typically employ fundamental electromagnetic or elastic energy relations to quantify the nonlinear dependence of the polarization $P$ on input fields $E$ [19]. These theories provide a framework for fundamentally quantifying material properties at the lattice level and may be necessary for optimal material design. However, for control applications, the large number of required parameters and states precludes real-time implementation using present hardware.

Macroscopic models are typically based on phenomenological principles, thermodynamic tenets, or energy formulations employed in concert with homogenization techniques. The former category includes Preisach models, which were originated for magnetic hysteresis [22], and have subsequently been extended to piezoceramic materials $[10,26]$. The advantage of Preisach theory lies in its generality and strong mathematical foundations which provide a framework for quantifying hysteresis when the underlying physics is poorly understood. However, the generality of the technique also yields models which have a large number of nonphysical parameters. Hence physical attributes of the data are difficult to utilize when identifying parameters or updating models to accommodate changing operating conditions although a number of extensions to the classical Preisach theory have recently been proposed to facilitate identification of parameters through correlation with physical principles $[8,20]$. Moreover, the original Preisach theory does not accommodate reversible effects or variable temperature, broadband operating conditions, and the modifications required to accommodate these effects can significantly diminish the efficiency of resulting models.

Macroscopic models based on energy techniques provide a compromise between microscopic or mesoscopic models and solely phenomenological models. Models in this category include the theory of Chen and Lynch [5], quasistatic hysteresis models of Huang and Tiersten [12] and the domain wall theory of Smith, Hom and Ounaies [32, 33]. While the underlying assumptions, specific formulations, and final goals differ in the respective approaches, similar strategies are employed when constructing models. In each case, energy relations are derived at the lattice or domain level and averaging techniques are invoked to obtain macroscopic models having a small number of effective parameters. For example, in the theory of [5], energy relations at the grain level are combined with macroscopic averaging over grain configurations to quantify strains and polarization in the aggregate material. In the domain wall theory of $[32,33]$, energy principles are employed to quantify local changes in polarization due to domain wall movement. These effects are then averaged to obtain macroscopic models for bulk material characterization and control design. 
The theory presented here is based on energy relations derived at the lattice or domain level with stochastic homogenization techniques employed to construct macroscopic models having a small number of effective parameters. In the first step of the development, Boltzmann principles are used to construct an expression for the Helmholtz energy through a balance of the internal energy due to positive or negative dipole configurations and entropy effects. The inclusion of the electrostatic work term provides a Gibbs relation which quantifies changes in the energy landscape due to an applied field. It is illustrated that minimization of the Gibbs energy yields a local polarization model that quantifies the phase transition from the ferroelectric to paraelectric state as temperatures are increased through the Curie point. Furthermore, this local relation is also the Ising model employed as an anhysteretic kernel in the domain wall theory of $[32,33]$. For implementation purposes, two asymptotic approximations are invoked: (i) a quadratic approximation to the Gibbs energy is constructed for fixed temperature regimes, and (ii) an algebraic model is constructed for the limiting case of negligible thermal activation. This provides a highly efficient technique for quantifying the $E-P$ relation in single crystal, homogeneous materials with uniform effective fields. In the final step of the model development, the effects of polycrystallinity, material nonhomogeneities, and nonuniform effective fields are incorporated by considering physical parameters such as the coercive and effective fields to be manifestations of stochastic distributions. This is motivated by the assumption that different domains have different energy characteristics, and it yields macroscopic models with parameters that effectively homogenize or average the material properties. It is illustrated through fits to experimental data that in spite of the incorporation of stochastic averages, several of the effective parameters can be directly correlated with physical properties of the data to aid parameter identification and model updating. It is also illustrated that the model enforces the deletion property and guarantees closure of both symmetric and asymmetric, biased minor loops. The model does not enforce congruency near saturation which reflects the observation that the measured $E-P$ response of the materials also does not exhibit congruency in these regions.

We note that while the specific motivation differs, analogous concepts involving stochastically distributed parameters are employed in Preisach formulations for magnetic compounds [8] and discrete models for shape memory alloys (SMA) based on elastic chains constructed from bi-stable elements $[23,24,25]$.

To place the theory in a broader context, we note that the free energy analysis used to construct the Helmholtz and Gibbs relations is an extension of the Müller-Achenbach-Seelecke theory for SMA $[21,29,30]$ whereas an analogous technique utilizing free energy relations in concert with stochastic distributions for the coercive and effective fields has been developed and implemented for ferromagnetic compounds [31]. Hence the technique provides a general methodology for quantifying hysteresis and constitutive nonlinearities inherent to a broad range of ferroic compounds [35]. Furthermore, it is illustrated in [37] that the theory provides an energy basis for Preisach models with two important differences: (i) the physical nature of parameters in the proposed model facilitates correlation with properties of the data, and (ii) temperature and relaxation dependencies are incorporated in the basis rather than in parameters as is the case for Preisach formulations. The latter property eliminates the necessity of vector-valued weights or lookup tables for material characterization and control design.

\section{Free Energies for Materials with Homogeneous Lattices}

Energy formulations for commonly employed ferroelectric materials can be motivated by changes which occur in the ionic structure during phase transitions and in response to input fields and stresses. To simplify the discussion, we will focus on barium titanate $\mathrm{BaTiO}_{3}$ and the piezoelectric compound $\mathrm{Pb}(\mathrm{Zr}, \mathrm{Ti}) \mathrm{O}_{3}$ or PZT. As detailed in [18], currently employed PZT transducer materials 
are comprised of $\mathrm{PbTi}_{1-x} \mathrm{O}_{3}$ and $\mathrm{PbZr}_{x} \mathrm{O}_{3}$ with $x$ chosen to optimize electromechanical coupling. The motivating discussion will emphasize $\mathrm{PbTiO}_{3}$ but analogous conclusions hold for $\mathrm{PbZrO}_{3}$ and $\mathrm{BaTiO}_{3}$.

These compounds are isostructural with the mineral perovskite $\left(\mathrm{CaTiO}_{3}\right)$ and exhibit what is termed a perovskite structure comprised of a cubic form at temperatures $T$ above the Curie point $T_{c}$ and a tetragonal form for $T<T_{c}$. We initially consider the idealized case of homogeneous materials having uniform lattices; hence for $T>T_{c}$, a unit cell at any point in the material will have the cubic ionic structure illustrated for $\mathrm{PbTi}_{1-x} \mathrm{O}_{3}$ in Figure 2a. At temperatures below $T_{c}$, the materials distorts from the cubic to tetragonal form through the biasing of $\mathrm{Ti}^{4+}$ ions toward $\mathrm{O}^{2-}$ pairs as illustrated for $\mathrm{BaTiO}_{3}$ on page 71 of [16]. In the absence of an applied electric field $E$, this ionic configuration produces a double well potential energy profile which varies as a function of the $\mathrm{Ti}^{4+}$ position. As depicted in Figure 3, the application of an electric field distorts the energy landscape and a dipole switch occurs when the equilibrium value determining the $\mathrm{Ti}^{4+}$ position exceeds the unstable equilibrium due to the central $\mathrm{O}^{2-}$ pairs. At the macroscopic scale, this produces a discontinuous jump in the polarization as experimentally illustrated for single crystal $\mathrm{BaTiO}_{3}$ on pages $72-76$ of [16].

\subsection{Helmholtz Energy}

We consider two techniques to quantify the Helmholtz free energy depicted in Figure 2c and 3b; the first is based on statistical mechanics principles and the second, while motivated by the first, is phenomenological.

Based on the assumption of material homogeneity, we consider a uniform lattice of volume $V$

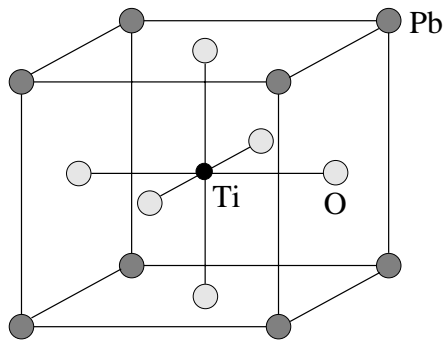

(a)

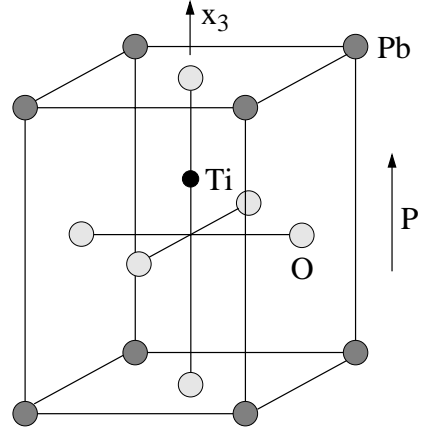

(b)

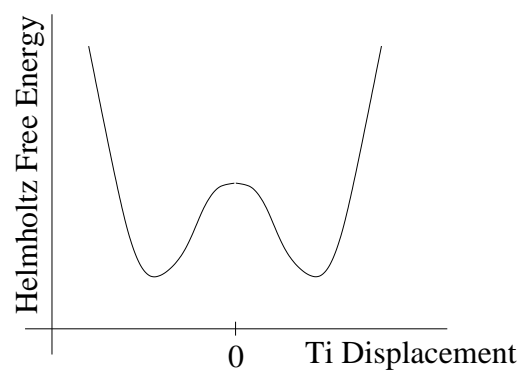

(c)

Figure 2. (a) Perovskite structure of $\mathrm{PbTiO}_{3}$ in the cubic form above $T_{c}$. (b) Tetragonal structure of $\mathrm{PbTiO}_{3}$ for $T<T_{c}$ and resulting spontaneous polarization. (c) Helmholtz free energy as a function of $\mathrm{Ti}$ position along the $x_{3}$-axis. 

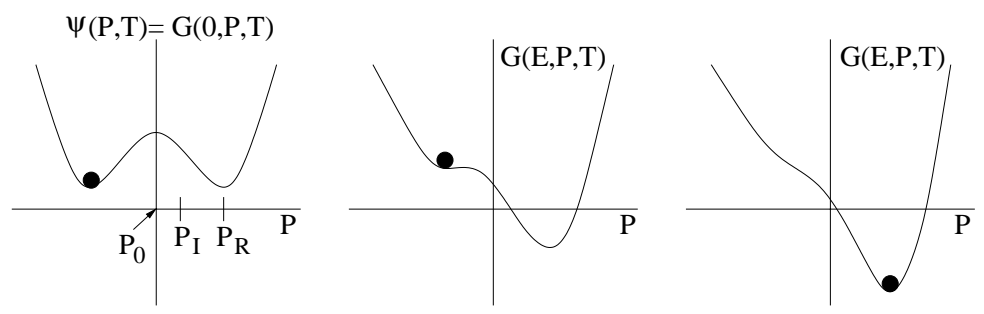

(a)
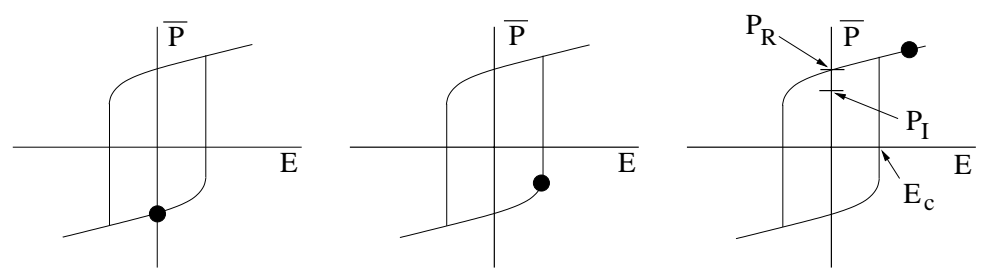

(b)

Figure 3. (a) Helmholtz energy $\psi$ and Gibbs energy $G$ for increasing field $E$. (b) Local polarization $\bar{P}$ as a function of $E$ for a homogeneous, isotropic material.

and mass $m$ having $N$ cells of the form depicted in Figure 2. Each cell is assumed to have dipole orientation $s_{i}= \pm 1$ and dipole moment $p_{0}$ so the polarization for the lattice is

$$
\begin{aligned}
P & =\frac{p_{0}}{V} \sum_{i=1}^{N} s_{i} \\
& =\frac{P_{s}}{N}\left(N_{+}-N_{-}\right) .
\end{aligned}
$$

Here $P_{s}=N p_{0} / V$ denotes the saturation polarization which occurs when all dipoles are positively aligned and $N_{+}$and $N_{-}$respectively denote the number of cells having positive and negative orientations. From the second equality in (1) and the fact that $N_{+}+N_{-}=N$, it follows that

$$
N_{+}=\frac{N}{2}\left(1+\frac{P}{P_{s}}\right) \quad, \quad N_{-}=\frac{N}{2}\left(1-\frac{P}{P_{s}}\right) .
$$

To compute the internal energy due to dipole reorientation, we let $\Phi_{0}$ denote the energy required to reorient a single dipole when the lattice is completely ordered $\left(P=P_{s}\right)$. For a general lattice ordering, the energies required to convert a dipole with positive orientation to negative, and conversely, are respectively

$$
\Phi_{+-}=\frac{N_{+}}{N} \Phi_{0} \quad, \quad \Phi_{-+}=\frac{N_{-}}{N} \Phi_{0} .
$$

We point out that these energy expressions are derived under the assumption that dipoles interact only with adjacent neighbors (e.g., see [11]).

The change in the internal energy due to dipole reorientation can then be expressed as

$$
d U=\left[\Phi_{+-} d N_{-}+\Phi_{-+} d N_{+}\right] \frac{1}{V} .
$$

By utilizing the relations (2) and (3), the expression (4) can be integrated to obtain the internal energy relation

$$
U=\frac{\Phi_{0} N}{2 V}\left(1-\frac{P^{2}}{P_{s}^{2}}\right)+U_{0}
$$


where $U_{0}$ denotes the energy for the completely ordered state. Since internal energy measures are relative, we take $U_{0}=0$ in subsequent relations.

The Helmholtz energy for the lattice is given by

$$
\psi=U-S T
$$

where $S$ denotes the entropy. From classical statistical mechanics arguments in combination with Stirling's formula (e.g., see [11]), the entropy is given by

$$
\begin{aligned}
S & =\frac{k}{V} \ln \left[\left(\begin{array}{c}
N \\
N_{+}
\end{array}\right)\right] \\
& =\frac{k}{V} \ln \left[\frac{N !}{N_{-} ! N_{+} !}\right] \\
& =\frac{k N}{V}\left[\ln 2-\frac{1+P / P_{s}}{2} \ln \left(1+\frac{P}{P_{s}}\right)-\frac{1-P / P_{s}}{2} \ln \left(1-\frac{P}{P_{s}}\right)\right] \\
& =\frac{-k N}{2 V P_{s}}\left[P \ln \left(\frac{P+P_{s}}{P_{s}-P}\right)+P_{s} \ln \left(1-\left(\frac{P}{P_{s}}\right)^{2}\right)\right]+S_{0}
\end{aligned}
$$

where $k$ denotes Boltzmann's constant and $S_{0}=\frac{k N}{V} \ln 2$. As with $U_{0}$, we neglect $S_{0}$ in the final relation for the Helmholtz energy since we are interested in a relative, rather than absolute, measure of energy.

The resulting Helmholtz free energy is

$$
\begin{aligned}
\psi(P, T) & =\frac{\Phi_{0} N}{2 V}\left[1-\left(P / P_{s}\right)^{2}\right]+\frac{T k N}{2 V P_{s}}\left[P \ln \left(\frac{P+P_{s}}{P_{s}-P}\right)+P_{s} \ln \left(1-\left(P / P_{s}\right)^{2}\right)\right] \\
& =\frac{E_{h} P_{s}}{2}\left[1-\left(P / P_{s}\right)^{2}\right]+\frac{E_{h} T}{2 T_{c}}\left[P \ln \left(\frac{P+P_{s}}{P_{s}-P}\right)+P_{s} \ln \left(1-\left(P / P_{s}\right)^{2}\right)\right] .
\end{aligned}
$$

In the second expression of (5), $E_{h}=\frac{N \Phi_{0}}{V P_{s}}$ is a bias field and $T_{c}=\frac{\Phi_{0}}{k}$ denotes the Curie temperature for the material. As illustrated in Figure 4, the relation (5) yields a double well potential at temperatures $T<T_{c}$ whereas behavior indicative of paraelectric materials is reflected by the single potential produced at $T>T_{c}$. However, the logarithmic nature of the entropic term reduces the efficiency of algorithms which employ this relation and makes it difficult to correlate parameters in the model with physically measured properties of the data.

A second technique for constructing the free energy, which addresses these difficulties, is based on the observation that for fixed temperatures, a first-order approximation to the Helmholtz relation (5) exhibits a quadratic dependence on the polarization in neighborhoods of all three equilibria. This motivates the piecewise quadratic definition

$$
\psi(P)= \begin{cases}\frac{1}{2} \eta\left(P+P_{R}\right)^{2} & , P \leq-P_{I} \\ \frac{1}{2} \eta\left(P-P_{R}\right)^{2} & , P \geq P_{I} \\ \frac{1}{2} \eta\left(P_{I}-P_{R}\right)\left(\frac{P^{2}}{P_{I}}-P_{R}\right) & ,|P|<P_{I}\end{cases}
$$

for the Helmholtz energy for fixed temperature regimes. As illustrated in Figure $3 \mathrm{a}, P_{I}$ and $P_{R}$ respectively denote the positive inflection point and polarization at which the minimum occurs. The relation between these points and local properties of the hysteresis kernel will be established in subsequent discussion. It will also be illustrated later that $\eta$ can be interpreted as the reciprocal slope $\frac{\partial E}{\partial P}$ of the hysteron in the post-switching linear regime. 


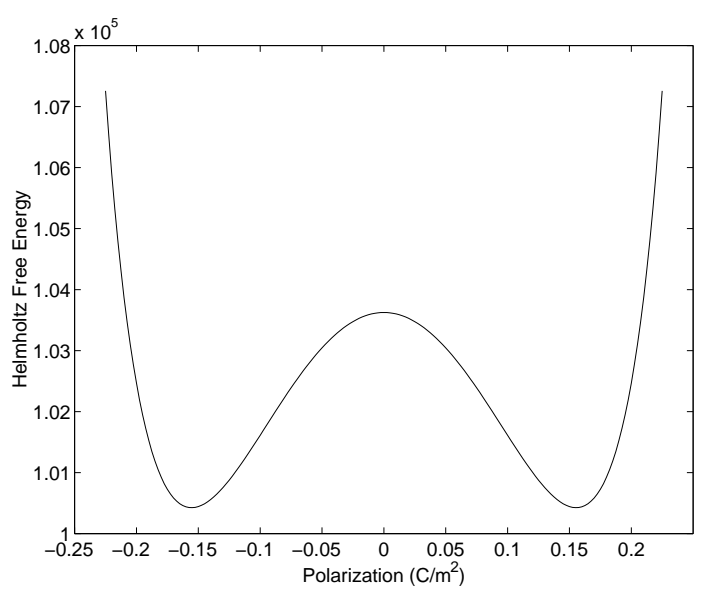

(a)

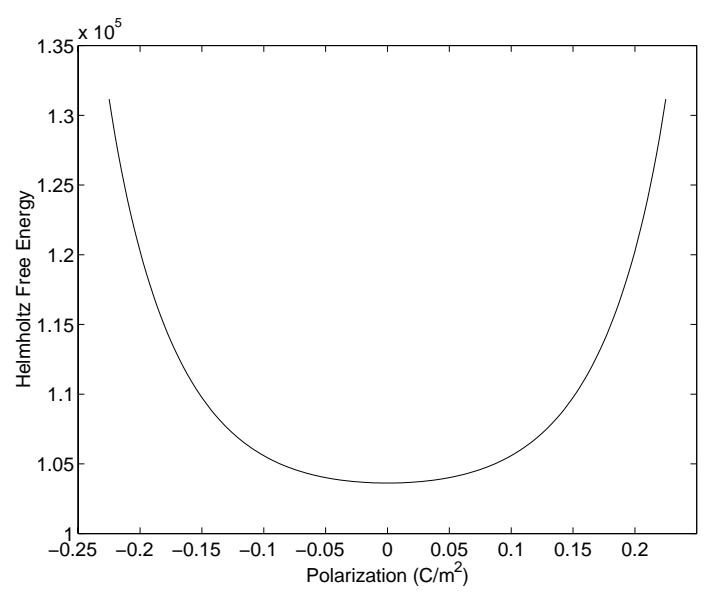

(b)

Figure 4. Helmholtz free energy specified by (5) for (a) $T<T_{c}$, and (b) $T>T_{c}$.

\subsection{Gibbs Energy}

To construct a Gibbs free energy which exhibits the behavior depicted in Figure 3a, the relation $U_{E}=-\mathbf{p} \cdot \mathbf{E}$ quantifying the potential energy of a dipole $\mathbf{p}$ in the field $\mathbf{E}$ is combined with the Helmholtz energy throughout the lattice to yield

$$
G=\psi-E P
$$

for $\psi$ given by (5) or (6). In the absence of applied stresses, the Gibbs relation (7) is used to characterize the energy landscape for homogeneous materials having a uniform lattice structure.

\subsection{Local Average Polarization}

The probability of obtaining the energy level $G$ for a lattice volume $V$ is specified through Boltzmann principles to be

$$
\mu(G)=C e^{-G V / k T}
$$

where $C$ is an integration constant chosen to yield a probability of 1 for integration over all admissible input values. For model identification, the volume $V$ is typically chosen to yield relaxation behavior appropriate for the material under consideration.

For a uniform input field $E$, the local average polarization at the lattice level is given by

$$
\bar{P}=x_{+}\left\langle P_{+}\right\rangle+x_{-}\left\langle P_{-}\right\rangle
$$

where $x_{+}$and $x_{-}$denote the fractions of dipoles having positive and negative orientations and $\left\langle P_{+}\right\rangle$ and $\left\langle P_{-}\right\rangle$are the expected polarization levels associated with positively and negatively oriented dipoles.

The evolution of the dipole fractions is specified by the differential equations

$$
\begin{aligned}
& \dot{x}_{+}=-p_{+-} x_{+}+p_{-+} x_{-} \\
& \dot{x}_{-}=-p_{-+} x_{-}+p_{+-} x_{+}
\end{aligned}
$$

which can be simplified to

$$
\dot{x}_{+}=-p_{+-} x_{+}+p_{-+}\left(1-x_{+}\right)
$$


through the identity $x_{+}+x_{-}=1$. Here $p_{+-}$denotes the likelihood of a dipole switching from positive to negative orientation whereas $p_{-+}$denotes the likelihood of switching from negative to positive (we avoid defining $p_{+-}$and $p_{-+}$as probabilities since they can be unbounded). The likelihoods are computed by specifying the probability of achieving the energy required for a jump multiplied by the frequency at which jumps are attempted. This yields the relations

$$
\begin{aligned}
& p_{+-}=\sqrt{\frac{k T}{2 \pi m V^{2 / 3}}} \frac{e^{-G\left(E, P_{0}(T), T\right) V / k T}}{\int_{P_{0}(T)}^{\infty} e^{-G(E, P, T) V / k T} d P} \\
& p_{-+}=\sqrt{\frac{k T}{2 \pi m V^{2 / 3}}} \frac{e^{-G\left(E,-P_{0}(T), T\right) V / k T}}{\int_{-\infty}^{P_{0}(T)} e^{-G(E, P, T) V / k T} d P}
\end{aligned}
$$

where $P_{0}(T)$ is the unstable equilibrium of $G$ and $m$ denotes the mass of the lattice. The denominator in both cases arises from evaluation of the integration constant $C$. When implementing the model, we typically replace $P_{0}(T)$ by the inflections points $P_{I}$ and $-P_{I}$, respectively, in the relations for $p_{+-}$and $p_{-+}$to obtain

$$
\begin{aligned}
& p_{+-}=\sqrt{\frac{k T}{2 \pi m V^{2 / 3}}} \frac{e^{-G\left(E, P_{I}, T\right) V / k T}}{\int_{P_{I}}^{\infty} e^{-G(E, P, T) V / k T} d P} \\
& p_{-+}=\sqrt{\frac{k T}{2 \pi m V^{2 / 3}}} \frac{e^{-G\left(E,-P_{I}, T\right) V / k T}}{\int_{-\infty}^{-P_{I}} e^{-G(E, P, T) V / k T} d P} .
\end{aligned}
$$

This simplifies the approximation of the integrals and can be motivated by observing that if one considers the forces $\frac{\partial G}{\partial P}$ due to the applied field, maximum restoring forces occur at $P_{I}$ and $-P_{I}$ (e.g., see pages 332-333 of [6]). Furthermore, for materials with low thermal activation, the points $P_{0}$ and $-P_{I}$ coincide in the limit as thermal activation is reduced to zero for positive fields while $P_{I}$ and $P_{0}$ coincide for negative fields as illustrated in Figure 5.

The expected polarizations are given by

$$
\left\langle P_{+}\right\rangle=\int_{P_{0}(T)}^{\infty} P \mu(G) d P \quad, \quad\left\langle P_{-}\right\rangle=\int_{-\infty}^{P_{0}(T)} P \mu(G) d P
$$

Specification of the probabilities using (8) and formulation of the integrals in terms of the inflection points yields the relations

$$
\left\langle P_{+}\right\rangle=\frac{\int_{P_{I}}^{\infty} P e^{-G(E, P, T) V / k T} d P}{\int_{P_{I}}^{\infty} e^{-G(E, P, T) V / k T} d P} \quad, \quad\left\langle P_{-}\right\rangle=\frac{\int_{-\infty}^{-P_{I}} P e^{-G(E, P) V / k T} d P}{\int_{-\infty}^{-P_{I}} e^{-G(E, P) V / k T} d P}
$$

quantifying the expected polarizations respectively due to positively and negatively oriented dipoles.

The summed products of the expectations and phase fractions (9) quantifies the local polarization $\bar{P}$ at the lattice level. For materials which are homogeneous and isotropic, this local polarization will be uniform throughout the material and hence it will also quantify the macroscopic polarization 


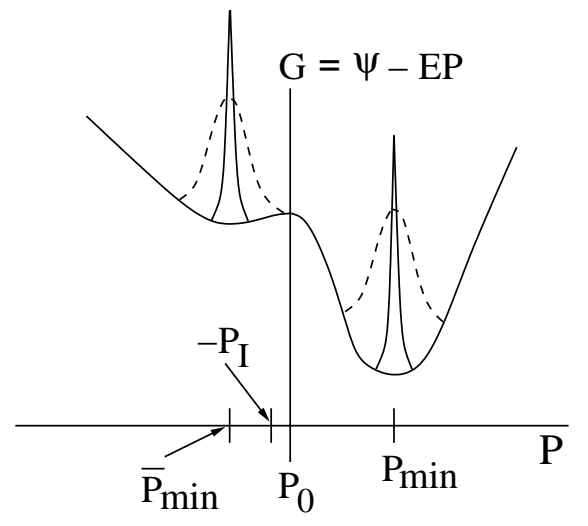

(a)

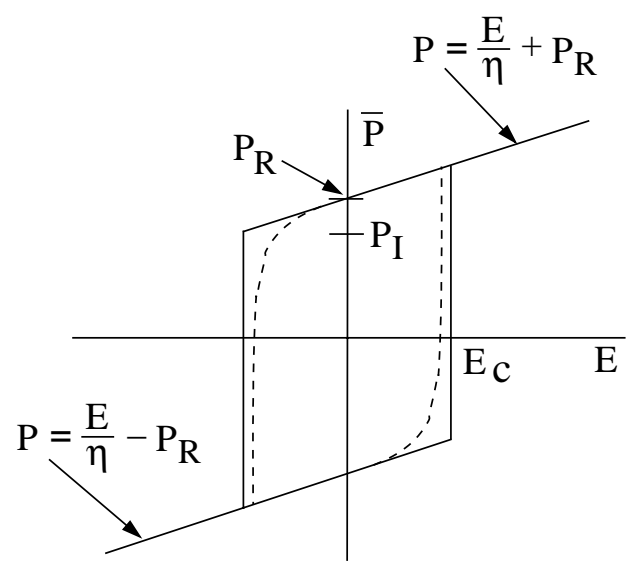

(b)

Figure 5. (a) Gibbs energy profile with high levels (-- ) and low levels $\left(-\frac{-}{-}\right)$ of thermal activation in the Boltzmann distribution $\mu(G)=C e^{-G V / k T}$. (b) Local polarization $\bar{P}$ given by equation (9) with high thermal activation $(---)$ and limiting polarization $\bar{P}$ specified by $(22)$ in the absence of thermal activation (-).

generated in response to an input field. With reasonable accuracy, this model could be used to quantify the single crystal $\mathrm{BaTiO}_{3}$ behavior depicted on pages 72-76 of [16]. Extensions to the model to accommodate nonhomogeneous material and field attributes will be provided in Section 3 .

The probabilistic nature of dipole switching produces the gradual transitions depicted in Figures $3 \mathrm{~b}$ and $5 \mathrm{~b}$, with the degree to which transitions are mollified being dependent on the ratio between $G V$ and $k T$ in the Boltzmann relation (8). Large values of $k T$, relative to $G V$, characterize regimes in which thermal activation is prominent which in turn produces smoother transitions since, for a fixed field level, dipoles have a higher probability of achieving the thermal energy required to overcome energy barriers.

For materials and operating regimes in which the relaxation effects due to thermal activation are negligible, the differential equation model which yields (9) can be simplified to a purely algebraic model through asymptotic analysis. This can improve the efficiency of model-based characterization and control algorithms and highlight additional properties of the local model.

As a prelude to this asymptotic analysis, we establish the following theorem.

Theorem 1. Let $f$ be continuous on the interval $[-L, L]$ and let $\left\{\phi_{j}\right\}$ be a sequence satisfying the following properties:

(i) $\phi_{j}>0$ for all $j$

(ii) $\int \phi_{j}(y) d y=1$ for all $j$

(iii) Given $\varepsilon, \delta>0$, there exists $j_{0}$ such that

$$
\int_{|y| \geq \delta} \phi_{j}(y)|f(y)| d y<\varepsilon / 3
$$

for all $j \geq j_{0}$. 
Then for $x \in[-L, L], \phi_{k} * f$ converges to $f$; that is

$$
\int \phi_{j}(x-y) f(y) d y \rightarrow f(x)
$$

Proof. From (ii) it follows that

$$
f(x)=f(x) \int \phi_{j}(y) d y=\int f(x) \phi_{j}(y) d y
$$

so that for $x \in[-L, L]$,

$$
\begin{aligned}
\phi_{j} * f(x)-f(x) & =\int \phi_{j}(y) f(x-y) d y-\int \phi_{j}(y) f(x) d y \\
& =\int \phi_{j}(y)[f(x-y)-f(x)] d y .
\end{aligned}
$$

Furthermore, from the continuity of $f$, it follows that for fixed $\varepsilon$, there exists $\delta$ such that

$$
|f(x-y)-f(x)|<\varepsilon / 3
$$

for $|y|<\delta$. For this $\delta$, we write

$$
\phi_{j} * f(x)-f(x)=\int_{|y|<\delta} \phi_{j}(y)[f(x-y)-f(x)] d y+\int_{|y| \geq \delta} \phi_{j}(y)[f(x-y)-f(x)] d y<\varepsilon
$$

for sufficiently large $j$. The integral over the region $|y|<\delta$ is bounded by $\varepsilon / 3$ due to the continuity of $f$ whereas the integral over $|y| \geq \delta$ is bounded by $2 \varepsilon / 3$ for sufficiently large $j$ as a result of (iii). The convergence follows directly.

We note that if we replace property (iii) by the requirement

$$
\int_{|y| \geq \delta} \phi_{j}(y) d y<\varepsilon
$$

and add the assumption that $f$ is measurable and bounded on $\mathbb{R}$, the sequence $\phi_{j}$ is termed a Dirac sequence on $\mathbb{R}$, and Theorem 1 is a $1-D$ version of Theorem 3.1 from page 228 of [13].

\subsection{Asymptotic Polarization Relation in Absence of Thermal Activation}

We now consider initial dipole fractions $\widehat{x}_{-}, \widehat{x}_{+}$, and a positive field $E$ for which $G\left(P_{\min }\right) \leq G\left(P_{0}\right)$ and $G\left(P_{\min }\right)<G\left(\bar{P}_{\min }\right)$ as depicted in Figure 5a. For simplicity, we consider the piecewise quadratic Helmholtz free energy model (6) and note that analogous asymptotic analysis holds for the statistical mechanics model (5). In this case, the minima

$$
\bar{P}_{\min }(E)=\frac{E}{\eta}-P_{R} \quad, \quad P_{\min }(E)=\frac{E}{\eta}+P_{R}
$$

result from the necessary condition $\frac{\partial G}{\partial P}=0$. The coercive field for which $\bar{P}_{\min }=P_{0}=-P_{I}$ is given by

$$
E_{c}=\eta\left(P_{R}-P_{I}\right) .
$$


We illustrate first that for $k T / V>0$, the Boltzmann probability (8) exhibits Gaussian behavior in neighborhoods of $\bar{P}_{\min }$ and $P_{\min }$ with variance proportional to $k T / V$. We will also illustrate that in the limit $k T / V \rightarrow 0, \mu(G)$ converges to a Dirac distribution.

For $P<-P_{I}, \mu(G)$ can be formulated as

$$
\begin{aligned}
\mu(G) & =\frac{e^{-\left[\frac{1}{2} \eta\left(P+P_{R}\right)^{2}-E P\right] V / k T}}{\int_{-\infty}^{-P_{I}} e^{-\left[\frac{1}{2} \eta\left(P+P_{R}\right)^{2}-E P\right] V / k T} d P} \\
& =\frac{e^{-\left(P-\bar{P}_{\text {min }}\right)^{2} \eta V / 2 k T}}{\int_{-\infty}^{-P_{I}} e^{-\left(P-\bar{P}_{\text {min }}\right)^{2} \eta V / 2 k T} d P} \\
& =\bar{C}(T, \beta) e^{-\left(P-\bar{P}_{\text {min }}\right)^{2} / 2 \beta^{2}}
\end{aligned}
$$

where

$$
\begin{aligned}
& \beta=\sqrt{\frac{k T}{\eta V}} \\
& \bar{C}(T, \beta)=\left[\int_{-\infty}^{-P_{I}} e^{-\left(P-\bar{P}_{\min }\right)^{2} / 2 \beta^{2}(T)} d P\right]^{-1} .
\end{aligned}
$$

We now let $j=1 / \beta$ and define the sequence

$$
\phi_{j}(P)=\left\{\begin{array}{cl}
\bar{C}(T, j) e^{-\left(P-\bar{P}_{\text {min }}\right)^{2} j^{2} / 2} & , \quad P \leq-P_{I} \\
0 & , \quad P>-P_{I} .
\end{array}\right.
$$

Since $\left\{\phi_{j}\right\}$ satisfies (i)-(iii) of Theorem 1, and hence constitutes a Dirac sequence, it follows that

$$
\begin{aligned}
\lim _{k T / V \rightarrow 0} \mu(G) & =\lim _{j \rightarrow \infty} \phi_{j}(P) \\
& =\delta\left(P-\bar{P}_{\text {min }}\right) .
\end{aligned}
$$

Analogous behavior is exhibited at $P_{\text {min }}$.

The Gaussian behavior of $\mu$, quantified by (15), is depicted in Figure 5a. From the definition of $\beta$, it follows that a decrease in thermal activation is reflected as a decrease in the variance. This implies that a smaller number of dipoles achieve the energy required to overcome the energy barrier which yields steeper transitions in the relation between $E$ and $\bar{P}$ as depicted in Figure 5b.

We now illustrate that the solution to the model (9) converges to the piecewise linear kernel specified by (13). It is first noted that as $k T / V \rightarrow 0$, the limiting solution to (10) is

$$
\begin{aligned}
x_{+} & =\widehat{x}_{+}+\left(1-\widehat{x}_{+}\right) h\left(P+P_{I}\right) \\
& =\left\{\begin{array}{cl}
\widehat{x}_{+}, & E<E_{c} \\
1, & E>E_{c}
\end{array}\right.
\end{aligned}
$$

where the local coercive field $E_{c}$ is given by (14) and $h$ denotes the Heaviside function. Corresponding values for $x_{-}$are determined through the relation $x_{+}+x_{-}=1$. 
The expected polarization due to positively oriented dipoles is

$$
\begin{aligned}
\left\langle P_{+}\right\rangle & =\lim _{k T / V \rightarrow 0} \frac{\int_{P_{I}}^{\infty} P e^{-G(E, P) V / k T} d P}{\int_{P_{I}}^{\infty} e^{-G(E, P) V / k T} d P} \\
& =\lim _{j \rightarrow \infty} \frac{\frac{j}{\sqrt{2 \pi}} \int_{P_{I}}^{\infty} P e^{-\left(P-P_{\min }\right)^{2} j^{2} / 2} d P}{\frac{j}{\sqrt{2 \pi}} \int_{P_{I}}^{\infty} e^{-\left(P-P_{\min }\right)^{2} j^{2} / 2} d P} \\
& =P_{\min }
\end{aligned}
$$

with the limits in the numerator and denominator evaluated using Theorem 1 with $f(P)=P$ and $f(P)=1$ for $P>P_{0}, f(P)=0$ for $P<P_{0}$, respectively. Similarly, the limiting value of $\left\langle P_{-}\right\rangle$is

$$
\left\langle P_{-}\right\rangle=\bar{P}_{\min } .
$$

From (18)-(22), it follows immediately that for the initial dipole distribution $\widehat{x}_{+}$and $\widehat{x}_{-}$, the local polarization is given by

$$
\bar{P}(E)= \begin{cases}\widehat{x}_{-} \bar{P}_{\min }(E)+\widehat{x}_{+} P_{\min }(E) & 0<E<E_{c} \\ P_{\min }(E) & E \geq E_{c}\end{cases}
$$

for positive fields $E$ as depicted in Figures 3 and 5 . Analogous results hold for negative fields.

To accommodate multiple transitions, the local polarization resulting from the Helmholtz relation (6) with no thermal activation can be formulated using Preisach notation (e.g., see [2, 37]) as

$$
\left[\bar{P}\left(E ; E_{c}, \xi\right)\right](t)= \begin{cases}{\left[\bar{P}\left(E ; E_{c}, \xi\right)\right](0)} & , \tau(t)=\emptyset \\ \frac{E}{\eta}-P_{R} & , \tau(t) \neq \emptyset \text { and } E(\max \tau(t))=-E_{c} \\ \frac{E}{\eta}+P_{R} & , \tau(t) \neq \emptyset \text { and } E(\max \tau(t))=E_{c}\end{cases}
$$

where

$$
\left[\bar{P}\left(E ; E_{c}, \xi\right)\right](0)= \begin{cases}\frac{E}{\eta}-P_{R} & , E(0) \leq-E_{c} \\ \xi & ,-E_{c}<E(0)<E_{c} \\ \frac{E}{\eta}+P_{R} & , E(0) \geq E_{c}\end{cases}
$$

denotes the dipole orientation yielding the initial polarization. A depiction of representative initial polarization values is provided in Figure 8. The transition times are designated as

$$
\tau(t)=\left\{t \in\left(0, T_{f}\right] \mid E(t)=-E_{c} \text { or } E(t)=E_{c}\right\}
$$

where $T_{f}$ denotes the final time under consideration. The dependence of $\bar{P}$ on the local coercive field $E_{c}$ defined in (14) is explicitly indicated since the parameter is considered to be distributed in the next section. We also note that the piecewise linear models (21) or (22) can be obtained directly from the necessary condition $\frac{\partial G}{\partial P}=0$; the asymptotic analysis illustrates the consistency of this condition in the limiting behavior of the full model (9). 


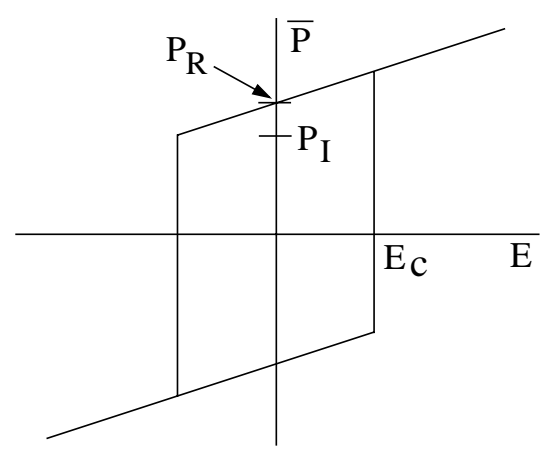

(a)

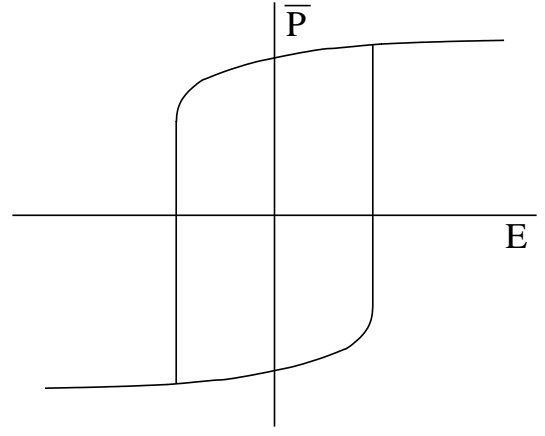

(b)

Figure 6. (a) Kernel provided by (22), and (b) kernel provided by (23).

Similar analysis can be used to specify the hysteresis kernel which results when $G$ is constructed using the Helmholtz relation (5) derived through statistical mechanics arguments. The determination of $\bar{P}$ through solution of $\frac{\partial G}{\partial P}=0$ in this case yields

$$
\begin{aligned}
\bar{P}\left(E ; E_{h}\right) & =P_{s} \tanh \left(\frac{E+E_{h} P / P_{s}}{E_{h} T / T_{c}}\right) \\
& =P_{s} \tanh \left(\frac{E+\alpha P}{a(T)}\right)
\end{aligned}
$$

where

$$
\alpha=\frac{E_{h}}{P_{s}} \quad, \quad a(T)=\frac{E_{h} T}{T_{c}} .
$$

The behavior of $\bar{P}$ specified by (23) is compared in Figure 6 with that defined by (21) or (22) which was derived from the piecewise quadratic Helmholtz energy. While the two representations yield similar kernel behavior as dipole switching occurs, the model (23) predicts a local saturation value of $P_{s}$ and decreasing slope for increasing field whereas the model (22) predicts linear behavior after dipole switching with slope $\frac{1}{\eta}$.

The Ising model (23) has been employed in a number of hysteresis models for ferroelectric materials and its ubiquity is due to the common underlying assumption that dipoles are aligned in only two configurations: in the direction of the applied field or diametrically opposite to it. As detailed in [32], quantification of the electrostatic energy under this assumption yields the Ising model whereas the relaxed assumption that dipoles can orient uniformly yields a Langevin model, which agrees with the Ising model through first-order terms but predicts a slower saturation rate. In both cases, these models were employed to quantify the anhysteretic kernel as an initial step in the development of a macroscopic hysteresis model $[32,33]$. The theory developed here differs from the domain wall theory in $[32,33]$ in the sense that the Ising model directly quantifies the energy landscape at the lattice level whereas it provides only an intermediate step in the domain wall theory. The saturation behavior of the Ising relation has also motivated its use in phenomenological models. Translates of the form $P=P_{s} \tanh \left(E \pm E_{c}\right)$ were employed by Zhang and Rogers [39], and $r(x)=\tanh (x)$ provides a suitable choice for the ridge functions employed in generalized Preisach, or Krasnolselskii-Pokrovskii, characterizations $[2,3]$. The model presented here differs in that it focuses on an energy formulation for domain processes. This yields a low-order model which, as illustrated in the examples, ensures closure of biased minor loops. 


\section{Polycrystalline Materials with Variable Effective Fields}

The local polarization relations (9), (22) and (23) were derived under the assumption of homogeneous and isotropic material properties and uniform effective fields $E_{e}=E$ throughout the materials. The consideration of the local relations throughout the material yields global models which predict instantaneous transitions at the coercive field as illustrated in Figures 4, 5 and 6 . While such global models can accurately quantify single crystal behavior of the type experimentally measured for $\mathrm{BaTiO}_{3}$ (e.g., see page 76 of [16]), they do not accurately predict the more gradual transition through the remanent polarization measured in polycrystalline ferroelectric materials. In this section, we incorporate the effects of nonuniform lattice configurations, polycrystallinity, and variable effective fields to provide a macroscopic model which accurately characterizes hysteresis in a variety of ferroelectric materials and ensures closure of biased minor loops.

\subsection{Distributions in Remanence Polarization, Lattice, and Coercive Field}

As illustrated in Figure 7, nonuniformities in the lattice produce a distribution of Helmholtz and Gibbs free energy profiles which can be manifested as variations in the local coercive field and local remanent polarization and can produce differing saturation behavior after dipole switching. Additional variations in the free energy relations will be produced by stress nonhomogeneities, nonuniform lattice orientations across grain boundaries, and crystalline anisotropies.

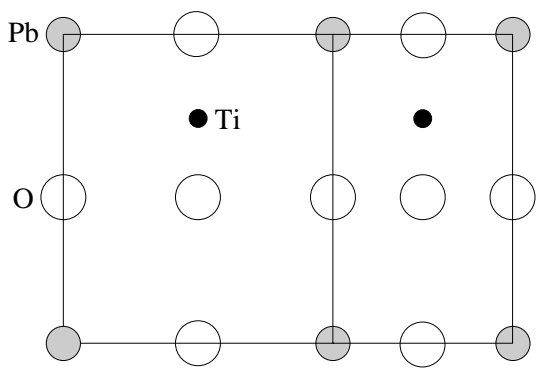

(i)

(ii)

(a)

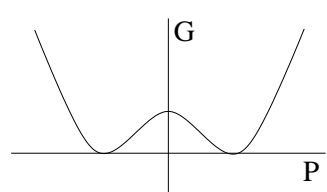

(i)

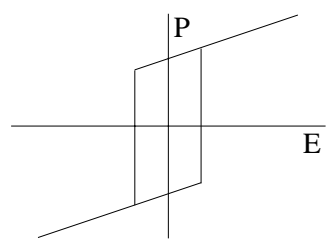

(i)

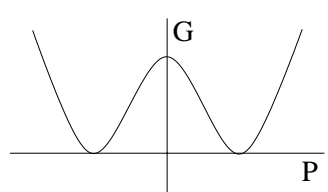

(ii)

(b)

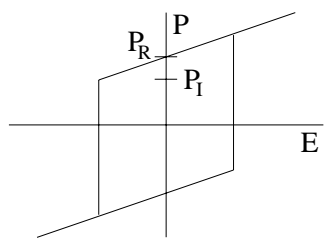

(c)

Figure 7. (a) Nonuniform lattice and polycrystalline structure for PZT; (b) Free energies associated with lattice widths (i) and (ii); (c) Variations in hysteresis kernel due to differing free energies. 
For the piecewise quadratic Helmholtz model (6), the variability in the lattice structure can be incorporated by considering $P_{R}, P_{I}$ or $E_{c}=\eta\left(P_{R}-P_{I}\right)$ to be manifestations of an underlying distribution rather than fixed values as assumed in the previous section for single crystals having uniform lattices. For this model, we consider variations in the local coercive field $E_{c}$ and make the assumption that it can be modeled by a log-normal distribution to incorporate the requirement that $E_{c}>0$. This yields the relation

$$
[P(E)](t)=\int_{0}^{\infty}\left[\bar{P}\left(E ; E_{c}, \xi\right)\right](t) f\left(E_{c}\right) d E_{c}
$$

for the macroscopic polarization where the density $f$ is specified by

$$
f\left(E_{c}\right)=c_{1} \exp \left\{-\left[\frac{\ln \left(E_{c} / \bar{E}_{c}\right)}{2 c}\right]^{2}\right\}
$$

and $\bar{P}$ is given by (9) or (22). Here $c, c_{1}$ and $\bar{E}_{c}$ are positive constants. In [8], it is illustrated that if $c$ is small compared with $\bar{E}_{c}$, the mean and variance have the approximate values

$$
\left\langle E_{c}\right\rangle \approx \bar{E}_{c} \quad, \quad \sigma \approx 2 \bar{E}_{c} c .
$$

As will be illustrated in the examples of Section 4, the relation (26) can be used to obtain initial parameter estimates based on attributes of measured experimental data. For materials in which the transition during hysteresis is relatively gradual, a second choice for $f$ is a normal distribution with mean $\bar{E}_{c}$ and variance $\sigma$. The lower integration limit of 0 should be retained to enforce nonnegative local coercive fields.

\subsection{Nonuniform Effective Fields}

The second extension employed to obtain a macroscopic model for the polarization entails the consideration of effective fields in the material. As noted in [1, 15, 32, 33], the applied field in ferroelectric materials is augmented by fields generated by neighboring dipoles which produce nonhomogeneous effective fields in the materials. This produces variations about the applied field which can significantly alter the measured polarization. To incorporate these field variations, we consider the effective field to be normally distributed about the applied field. For fixed $E_{c}$, the polarization in this case is given by

$$
[P(E)](t)=\int_{-\infty}^{\infty} c_{2}\left[\bar{P}\left(E_{e} ; E_{c}, \xi\right)\right](t) e^{-\left(E-E_{e}\right)^{2} / b} d E_{e} .
$$

The introduction of variations in the effective field produces domain switching in advance of the remanence point in accordance with observations from experimental data.

The complete macroscopic polarization model for nonhomogeneous, polycrystalline materials with variable effective fields, as based on the piecewise quadratic Helmholtz model (6), is then given by

$$
[P(E)](t)=C \int_{0}^{\infty} \int_{-\infty}^{\infty}\left[\bar{P}\left(E_{e}+E, E_{c}, \xi\right)\right](t) e^{-E_{e}^{2} / b} e^{-\left[\ln \left(E_{c} / \bar{E}_{c}\right) / 2 c\right]^{2}} d E_{e} d E_{c}
$$

where $\bar{P}$ is specified by (9) or (22). We note that while the model (28) incorporates certain relaxation mechanisms, it does not incorporate dynamic elastic effects. Hence this formulation of the polarization model should be restricted to low frequency drive regimes. 
Similar arguments can be employed to construct a macroscopic polarization model based on the Helmholtz free energy relation (5) obtained through statistical mechanics analysis. In this case, we assume that the bias field $E_{h}$ is a manifestation of an underlying log-normal distribution to obtain the global relation

$$
[P(E)](t)=C \int_{0}^{\infty} \int_{-\infty}^{\infty}\left[\bar{P}\left(E_{e}+E, E_{h}, \xi\right)\right](t) e^{-E_{e}^{2} / b} e^{-\left[\ln \left(E_{h} / \bar{E}_{h}\right) / 2 c\right]^{2}} d E_{e} d E_{h} .
$$

Here $\bar{P}$ is specified by (9) or (23).

The polarization behavior predicted by (29) differs from that of (28) primarily at high input field levels. The E-P behavior predicted by (28) reflects the linear behavior associated with the hysteresis kernel (22) after completion of dipole switching whereas the $E$ - $P$ curve produced by (29) saturates to zero slope due to the behavior of the hyperbolic tangent kernels. While both models are appropriate for a number of materials, the saturation behavior and ease with which the respective models can be implemented are effective criteria for choosing between the models (28) and (29) for a given application.

\subsection{Implementation Issues}

To implement the models (28) or (29), it is necessary to approximate the integrals. This can be accomplished on the semi-infinite domain using Gauss-Laguerre quadrature and on the infinite domain using Gauss-Hermite quadrature (e.g., see pages 698-699 of [40]). Alternatively, the exponential decay of the kernels can be employed to truncate the domains to finite intervals appropriate for Gauss-Legendre formulae (see Figure 8a). In all cases, approximation of (28) yields expressions of the form

$$
[P(E)](t)=C \sum_{i=1}^{N_{i}} \sum_{j=1}^{N_{j}}\left[\bar{P}\left(E_{e_{j}}+E ; E_{c_{i}}, \xi_{i}\right)\right](t) e^{-E_{e_{j}}^{2} / b} e^{-\left[\ln \left(E_{c_{i}} / E_{c}\right) / 2 c\right]^{2}} v_{i} w_{j}
$$

with a similar relation resulting from the approximation of (29). Here $E_{e_{j}}, E_{c_{i}}$ denote the abscissas associated with respective quadrature formulae and $v_{i}, w_{j}$ are the respective weights.

For the examples reported in Section 4, we employed composite Gauss-Legendre quadrature on truncated intervals chosen to be commensurate with nontrivial values of the integrands. To illustrate, we provide the abscissas and weights employed in the approximation of the integral (27) on the truncated domain $[-L, L]$ using a four point composite quadrature rule. Letting $h_{j}=-L+j h$, the quadrature points and weights on each subinterval $\left[h_{j-1}, h_{j}\right]$ are

$$
\begin{array}{lll}
E_{e_{j 1}}=h_{j-1}+h\left[\frac{1}{2}-\frac{\sqrt{15+2 \sqrt{30}}}{2 \sqrt{35}}\right] & & w_{j 1}=\frac{49 h}{12(18+\sqrt{30})} \\
E_{e_{j 2}}=h_{j-1}+h\left[\frac{1}{2}-\frac{\sqrt{15-2 \sqrt{30}}}{2 \sqrt{35}}\right] & , & w_{j 2}=\frac{49 h}{12(18-\sqrt{30})} \\
E_{e_{j 3}}=h_{j-1}+h\left[\frac{1}{2}+\frac{\sqrt{15-2 \sqrt{30}}}{2 \sqrt{35}}\right] & & w_{j 3}=\frac{49 h}{12(18-\sqrt{30})} \\
E_{e_{j 4}}=h_{j-1}+h\left[\frac{1}{2}+\frac{\sqrt{15+2 \sqrt{30}}}{2 \sqrt{35}}\right] & & w_{j 4}=\frac{49 h}{12(18+\sqrt{30})} .
\end{array}
$$

The quadrature points and initial polarization values $\xi_{j}$ for $E=0, P=0$, with the hysteresis kernel (22), are depicted for $N_{q}=2\left(N_{j}=8\right)$ in Figure $8 \mathrm{~b}$. 


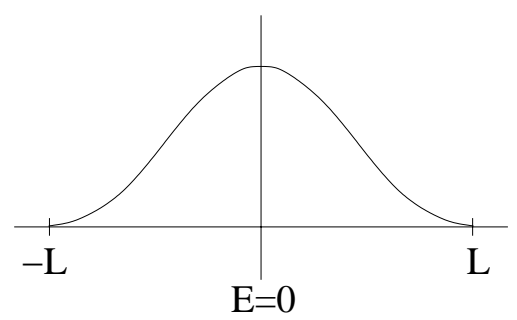

(a)

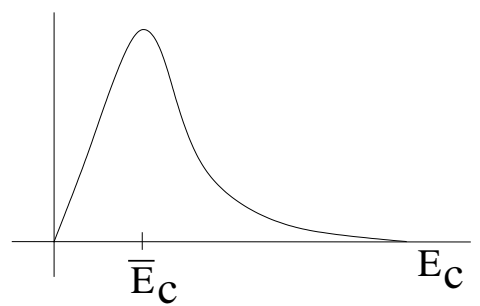

(c)

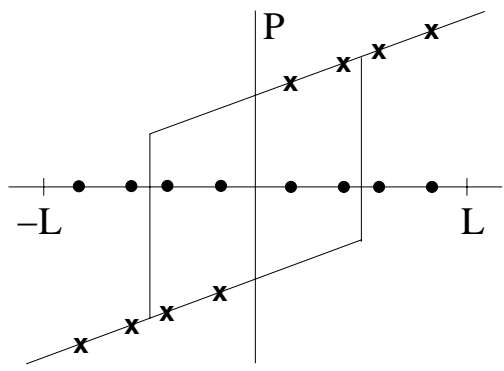

(b)

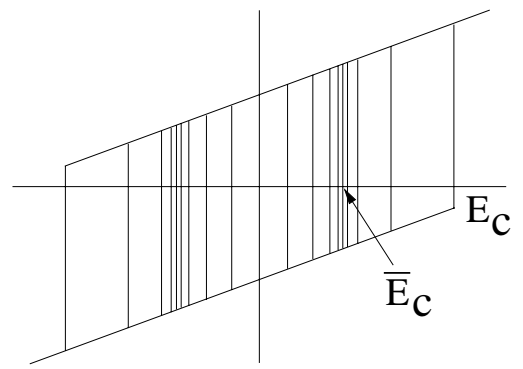

(d)

Figure 8. (a) Decay exhibited by $f_{2}\left(E_{e}\right)=e^{-E_{e}^{2} / b}$ and truncated domain $[-L, L]$. (b) Gaussian quadrature points $\bullet$ and initial local polarization values $\xi_{j}$ (indicated by $\mathbf{x}$ ) for $N_{j}=8$. (c) $\log$ normal density $f\left(E_{c}\right)=c_{1} e^{-\left[\ln \left(E_{c} / \bar{E}_{c}\right) / 2 c\right]^{2}}$ having mean $\bar{E}_{c}$. (d) Distribution of hysteresis kernels having coercive fields $E_{c}$.

A second implementation issue concerns the manner through which the conditional definitions in (22) are evaluated if this kernel is employed in the hysteresis model. While it is algorithmically straightforward to implement these conditions using the transition times $\tau(t)$, this must be done for all quadrature points $E_{e_{j}}$ and $E_{c_{i}}$ for each input field value. Implementation in this manner significantly diminishes the speed of the model evaluation and would likely prohibit the use of the model for real-time control design and implementation.

An alternative is to formulate the local polarization (22) as

$$
\bar{P}=\frac{E}{\eta}+P_{R} \Delta
$$

where $\Delta=1$ if evaluating on the upper branch of the kernel and $\Delta=-1$ if evaluating on the lower branch. The crux of the algorithm focuses on the efficient construction of $\Delta$ which accommodates the vector nature of the effective field values and coercive fields resulting from the quadrature of the integrals. Intuitively, the local polarization values associated with each effective field value $E_{e_{j}}$ will jump when they reach a coercive field $E_{c_{i}}$. Because both the effective and coercive field values are distributed, as depicted in Figure 8, this leads to $N_{i} \times N_{j}$ relations which must be checked for each input field value $E$. Hence for the distributed algorithm, $\Delta$ is an $N_{i} \times N_{j}$ matrix in which the $i j$ th element specifies whether the $j$ th effective field value $E_{e_{j}}$ has crossed the $i$ th coercive value $E_{c_{i}}$ to determine whether the associated polarization value is on the upper or lower branch of the hysteron. The high efficiency of the algorithm is achieved by employing algebraic matrix operations to construct $\Delta$ rather than conditional statements implemented through if-then constructs. 
To illustrate the algorithm used to compute $P$ using the approximate relation (30), with $\bar{P}$ given by (22), we first define the following matrices

$$
\begin{gathered}
\Delta_{\text {init }}=\left[\begin{array}{rrrrrr}
-1 & \cdots & -1 & 1 & \cdots & 1 \\
\vdots & & \vdots & \vdots & & \vdots \\
-1 & \cdots & -1 & 1 & \cdots & 1
\end{array}\right]_{N_{i} \times N_{j}} \quad, \quad \mathcal{E}_{c}=\left[\begin{array}{lll}
E_{c_{1}} & \cdots & E_{c_{1}} \\
\vdots & & \vdots \\
E_{c_{N_{i}}} & \cdots & E_{c_{N_{i}}}
\end{array}\right]_{N_{i} \times N_{j}} \\
\mathcal{E}_{k}=\left[\begin{array}{lll}
E_{k}+E_{e_{1}} & \cdots & E_{k}+E_{e_{N_{j}}} \\
\vdots & & \vdots \\
E_{k}+E_{e_{1}} & \cdots & E_{k}+E_{e_{N_{j}}}
\end{array}\right]_{N_{i} \times N_{j}} \quad, \quad \mathcal{O}=\left[\begin{array}{lll}
1 & \cdots & 1 \\
\vdots & & \vdots \\
1 & \cdots & 1
\end{array}\right]_{N_{i} \times N_{j}}
\end{gathered}
$$

and weight vectors

$$
\begin{aligned}
& W^{T}=\left[w_{1} e^{-E_{e_{1}}^{2} / b}, \cdots, w_{N_{j}} e^{-E_{e_{N_{j}}}^{2} / b}\right]_{1 \times N_{j}} \\
& V^{T}=\left[v_{1} e^{-\left[\ln \left(E_{c_{1}} / \bar{E}_{c}\right) / 2 c\right]^{2}}, \cdots, v_{N_{i}} e^{-\left[\ln \left(E_{c_{N_{i}}} / \bar{E}_{c}\right) / 2 c\right]^{2}}\right]_{1 \times N_{i}}
\end{aligned}
$$

where $E_{k}=E\left(t_{k}\right)$ is the $k$ th value of the input field. The polarization $P_{k} \approx P\left(E_{k}\right)$ is then specified by the following algorithm.

\section{Algorithm 1.}

$$
\begin{aligned}
& \Delta=\Delta_{\text {init }} \\
& \widehat{P}=\frac{1}{\eta} \mathcal{E}_{c}+P_{R} \mathcal{O} \\
& \text { for } k=1: N_{k} \\
& \qquad \bar{P}=\frac{1}{\eta} \mathcal{E}_{k}+P_{R} \Delta \\
& \quad d E=E_{k}-E_{k-1} \\
& \qquad \begin{aligned}
\Delta & \operatorname{sgn}\left(\left(\mathcal{E}_{k}-\operatorname{sgn}(d E) \mathcal{E}_{c}\right) \cdot *(\bar{P}-\operatorname{sgn}(d E) \widehat{P}) \cdot * \bar{P}\right) \\
& \bar{P}=\frac{1}{\eta} \mathcal{E}_{k}+P_{R} \Delta \\
& P_{k}=C V^{T} \bar{P} W
\end{aligned}
\end{aligned}
$$

end

In this algorithm, .* indicates componentwise matrix multiplication and sgn denotes the signum function. Depending on the methods used for programming, the use of Algorithm 1 rather than utilizing conditional if-then constructs can reduce runtimes by factors in excess of 100 so that full multiloop model simulations run in the order of seconds on a workstation. This level of efficiency is necessary to achieve real-time implementation of control algorithms utilizing this model.

\section{Model Validation}

To illustrate attributes of the model, we consider two sets of examples. In the first, the capability of the model to characterize symmetric, major loop properties of PZT5A, PZT5H and PZT4 is illustrated through comparison with experimental data. For each compound, model parameters 
are estimated through a least squares fit to data at high drive levels and the resulting model is used to predict the model behavior in lower drive regimes. In all cases, quasistatic drive regimes are considered. The second example illustrates numerically the capability of the model to quantify biased minor loop behavior including Raleigh loops at low drive levels and multiply nested loops at intermediate levels. In concert, these examples illustrate the flexibility of the model for a variety of materials and operating conditions.

\subsection{Determination of Parameters}

The continuous model (28) or discretized model (30) contains the parameters $P_{R}, \eta, b, \bar{E}_{c}, c$ and $C$ which must be specified when quantifying a specific PZT compound. Similar parameters arise in the model derived through statistical mechanics principles. Asymptotic analysis can be employed to obtain initial parameter choice which can then be employed in various least squares formulations to determine parameters which optimize model fits and predictions.

As illustrated in Figure 5, $P_{R}$ denotes the local remanence polarization for a domain and $\eta$ is the reciprocal of the slope in the $E-P$ relation after switching. The inclusion of polycrystallinity, variable effective fields and material nonhomogeneities through the density analysis in Section 3 makes it difficult to correlate the remanence polarization measured for the bulk material with the local value $P_{R}$; hence $P_{R}$ is typically estimated solely through a least squares fit to the data. Moreover, for the linear relations (22) or (31) for the kernel $\bar{P}, P_{R}$ and $C$ produce analogous scaling in the bulk polarization so that they can be combined when estimating parameters. The slope of the local kernel relations scales through the stochastic homogenization process so bulk measurements of the reciprocal slope $\frac{d E}{d P}$ provide initial estimates for $\eta$. The distribution $f$, defined in (25), quantifies

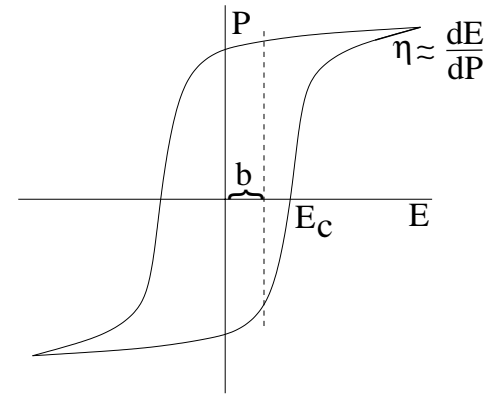

(a)

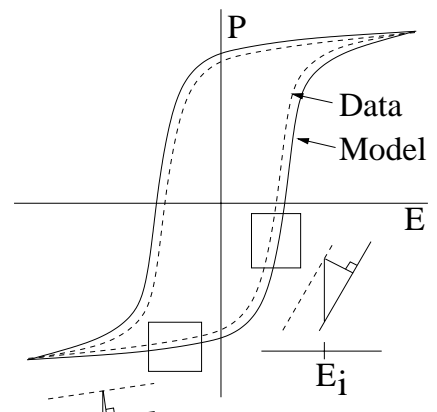

(b)

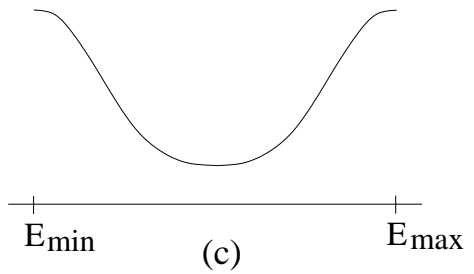

Figure 9. (a) Asymptotic relations between the parameters $\eta, \bar{E}_{c}, b$ and the slope of the $P$ - $E$ relation after switching, the coercive field and the point where switching commences before remanence. (b) The absolute metric (32) and Euclidean metric (34) between data and the model prediction. (c) Cosine filter (33) used to minimize least squares differences in the switching region. 
the coercive properties of the $E-P$ relation. The mean $\bar{E}_{c}$ quantifies the point at which the primary switching occurs as illustrated in Figure $9 \mathrm{a}$; hence $\bar{E}_{c}$ is asymptotically given by the coercive field for the bulk material. The variance $\sigma \approx 2 \bar{E}_{c} c$ quantifies the variability in local coercive fields so that materials with steep coercive transitions yield small values of $c$ whereas large parameter values are required when characterizing materials with gradual bulk switching. The parameter $b$ quantifies the variance in the effective field which determines, in part, the degree to which switching occurs before remanence is reached. Materials with nearly linear $E-P$ relations at remanence yield small values of $b$ whereas large values are required to accommodate significant switching before remanence.

Hence physical properties of the data yield initial estimates for $\eta$ and $\bar{E}_{c}$ and provide qualitative techniques for ascertaining $b, c, P_{R}$ and $C$. This significantly facilitates the implementation of least squares techniques used to determine model parameters and update these parameters to accommodate slowly changing operating conditions.

Three least squares techniques have been considered to accommodate the switching and saturation behavior inherent to hysteresis data. To formulate the least squares problems, let $\left(\widehat{E}_{i}, \widehat{P}_{i}\right), i=$ $1, \cdots, \mathcal{N}$, denote the field and corresponding polarization data measured throughout the hysteresis cycle. Furthermore, let $P\left(\widehat{E}_{i} ; q\right)$ denote parameter-dependent model solutions provided by $(28),(29)$ or (30). For admissible parameters $q \in Q$, we then consider the following optimization problems:

$$
\begin{gathered}
\min _{q} \sum_{i=1}^{\mathcal{N}}\left|P\left(\widehat{E}_{i} ; q\right)-\widehat{P}_{i}\right|^{2} \\
\min _{q} \sum_{i=1}^{\mathcal{N}} \mu_{i}\left|P\left(\widehat{E}_{i} ; q\right)-\widehat{P}_{i}\right|^{2}, \quad \mu_{i} \equiv \cos \left[2 \pi \cdot \frac{\widehat{E}_{i}-E_{\text {min }}}{E_{\max }-E_{\min }}\right]+\alpha \\
\min _{q} \sum_{i=1}^{\mathcal{N}} d\left(P\left(\widehat{E}_{i} ; q\right)-\widehat{P}_{i}\right) .
\end{gathered}
$$

The functional (32) penalizes absolute differences between the data and model. As illustrated in Figure 9b, it will produce model fits which tend to be more accurate in the high gradient switching regime than in the low gradient saturation region. For applications which require high accuracy throughout the drive range, two techniques can be employed to modify the manner through which the difference between the model and data are penalized. One alternative is to employ the functional (33) which weights the data in the saturation region more heavily through a cosine filter of the type illustrated in Figure 9c. A more accurate, but computationally more intensive, technique employs the Euclidean metric $d$ which minizes the distance between the model and data as illustrated in Figure $9 \mathrm{~b}$. For each functional, initial parameter choices $q_{0}$ are obtained through the previously discussed asymptotic relations or a priori material information.

\subsection{Experimental Validation for PZT5A, PZT5H and PZT4}

To illustrate the accuracy and flexibility of the model and parameter estimation techniques for a variety of compounds, we consider the characterization of PZT5A, PZT5H and PZT4 wafers. In all cases, data was collected at $200 \mathrm{mHz}$ to minimize frequency effects. For consistency, the discretized model (30), with $\bar{P}$ given by (22) or (31), was employed in all three cases. However, we note that analogous results have been obtained with the kernel (9) and the discretized version of the statistical mechanics model (29). Finally, the limits $N_{i}=N_{j}=80$ in (30) - obtained using $N_{q}=N_{p}=20$ subdivisions with the 4 point Gaussian rule - ensured convergence of the Gaussian quadrature routines. 


\section{PZT5A}

We consider first the characterization of hysteresis exhibited by PZT5A for various input field levels. Data was collected from a rectangular $1.7 \mathrm{~cm} \times 0.635 \mathrm{~cm} \times 0.0381 \mathrm{~cm}$ wafer at peak voltages ranging from $600 \mathrm{~V}$ to $1600 \mathrm{~V}$. Corresponding field values were computed using the relation

$$
E=V / h
$$

where $h=3.81 \times 10^{-4} \mathrm{~m}$ denotes the thickness of the wafer. The resulting hysteretic $E$ - $P$ relations are plotted in Figure 10.

The polarization was modeled using the relation (30) with the piecewise linear kernel (22). The coercive field for the $1600 \mathrm{~V}$ data is $1.2 \times 10^{6} \mathrm{~V} / \mathrm{m}$ and the slope after field reversal in saturation is $3.6 \times 10^{8}$. These two values were respectively employed as initial estimates for $\bar{E}_{c}$ and $\eta$. The functional (32) was then employed to estimate the parameters $P_{R}=0.04 \mathrm{C} / \mathrm{m}^{2}, \bar{E}_{c}=0.96507 \times$ $10^{6} \mathrm{~V} / \mathrm{m}, \eta=9 \times 10^{8}, c=0.3582 \mathrm{~V}^{2} / \mathrm{m}^{2}, b=2.1407 \times 10^{11} \mathrm{~V}^{2} / \mathrm{m}^{2}$ and $C=8.57 \times 10^{-12}$ through a fit to the $1600 \mathrm{~V}$ data. The model with these parameter values was then used to predict the hysteretic $E-P$ relation at the $600 \mathrm{~V}, 800 \mathrm{~V}$ and $1000 \mathrm{~V}$ input levels yielding the fits plotted in Figure 10. It is observed that the model accurately quantifies the hysteresis through the switching region at $1600 \mathrm{~V}$ but is less accurate near saturation since errors in this region are penalized less by the absolute functional (32). The accuracy of the predictions at the lower drive levels attests to the flexibility of the model for quantifying the $E-P$ relation through a wide range of field inputs.

The use of the cosine-weighted functional (33) yields the parameters $P_{R}=0.04 \mathrm{C} / \mathrm{m}^{2}, \bar{E}_{c}=$ $0.866010 \times 10^{6} \mathrm{~V} / \mathrm{m}, \eta=9.5 \times 10^{8}, c=0.4272 \mathrm{~V}^{2} / \mathrm{m}^{2}, b=1.9754 \times 10^{11} \mathrm{~V}^{2} / \mathrm{m}^{2}$ and $C=7.9926 \times 10^{-12}$ and produces a model fit with slightly improved accuracy in the saturation region but less accuracy near the coercive field and at low drive levels (see Figure 11). Both algorithms provide sufficient accuracy for quantifying hysteresis inherent to PZT5A for a broad range of applications.
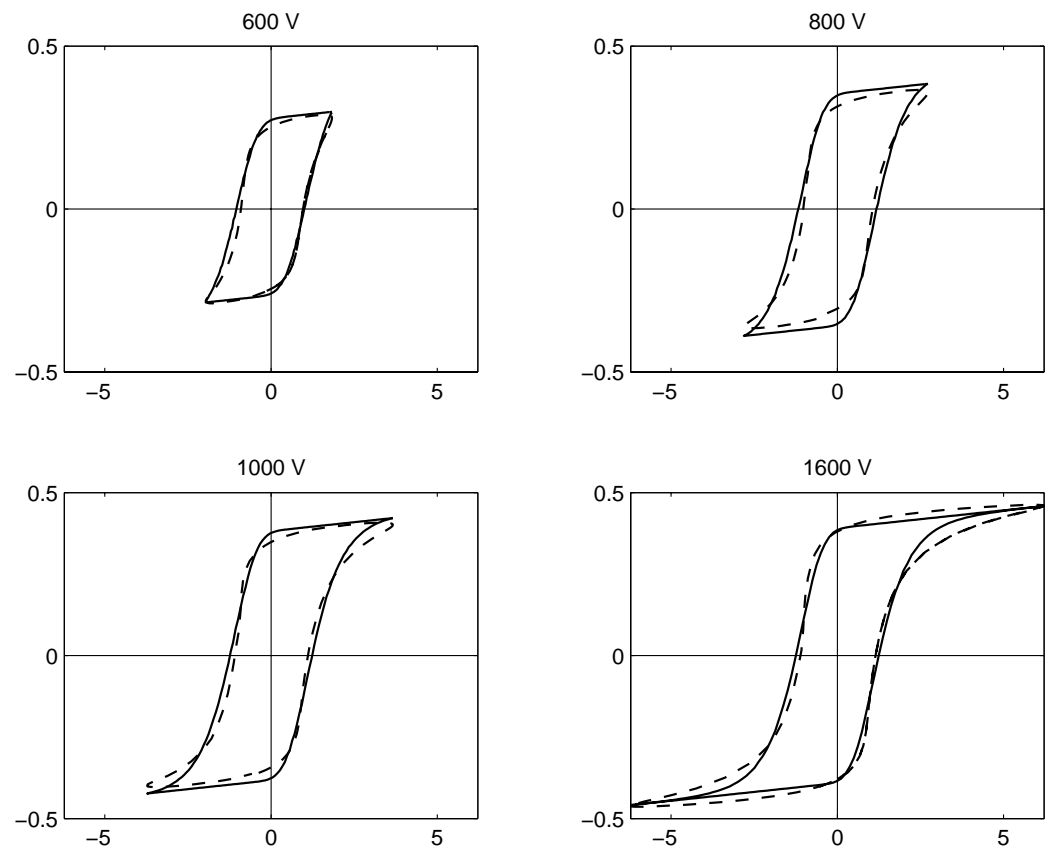

Figure 10. PZT5A data (-- ) and model predictions (-) with the parameters $P_{R}=0.04 \mathrm{C} / \mathrm{m}^{2}$, $\bar{E}_{c}=0.96507 \times 10^{6} \mathrm{~V} / \mathrm{m}, \eta=9 \times 10^{8}, c=0.3582 \mathrm{~V}^{2} / \mathrm{m}^{2}, b=2.1407 \times 10^{11} \mathrm{~V}^{2} / \mathrm{m}^{2}, C=8.57 \times 10^{-12}$ determined by the absolute least squares functional (32). Abscissas: electric field $(\mathrm{MV} / \mathrm{m})$, ordinates: polarization $\left(\mathrm{C} / \mathrm{m}^{2}\right)$. 

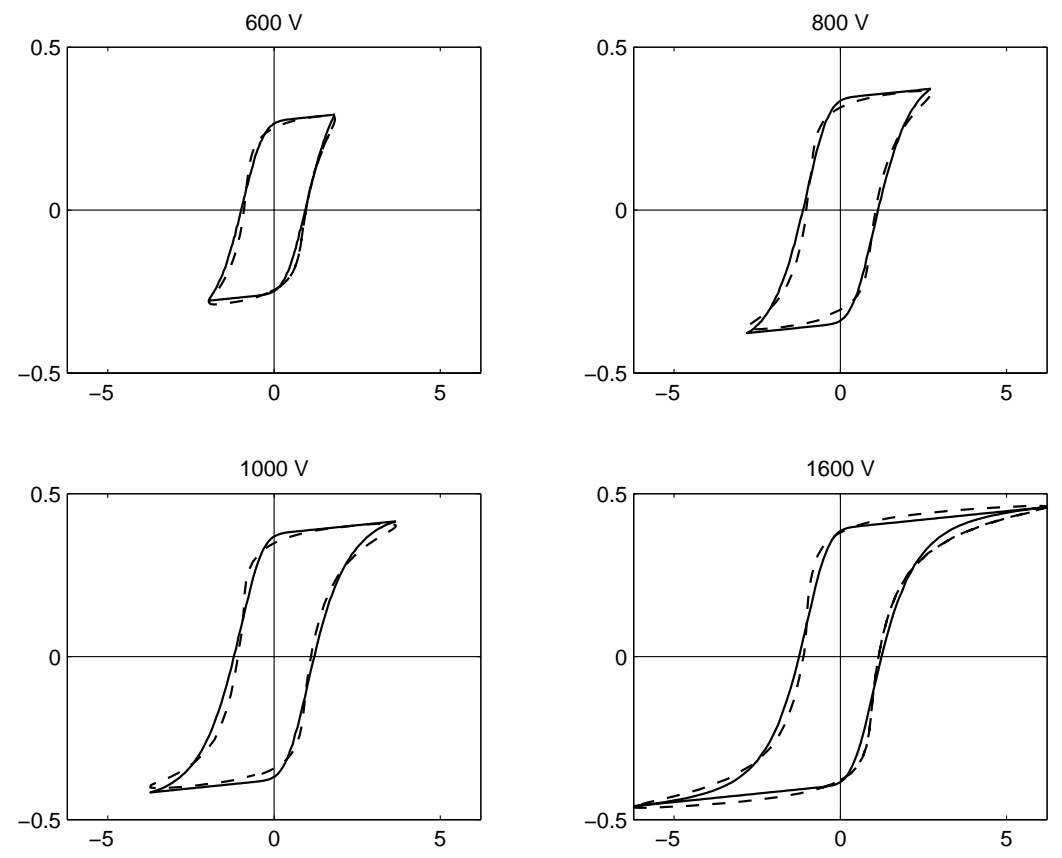

Figure 11. PZT5A data (-- ) and model predictions (-) with the parameters $P_{R}=0.04 \mathrm{C} / \mathrm{m}^{2}$, $\bar{E}_{c}=0.866010 \times 10^{6} \mathrm{~V} / \mathrm{m}, \eta=9.5 \times 10^{8}, c=0.4272 \mathrm{~V}^{2} / \mathrm{m}^{2}, b=1.9754 \times 10^{11} \mathrm{~V}^{2} / \mathrm{m}^{2}, C=$ $7.9926 \times 10^{-12}$ determined by the cosine-weighted least squares functional (33). Abscissas: electric field $(\mathrm{MV} / \mathrm{m})$, ordinates: polarization $\left(\mathrm{C} / \mathrm{m}^{2}\right)$.

\section{PZT5H}

To illustrate the performance of the model for characterizing a second soft PZT compound, we consider data collected from a $3.81 \mathrm{~cm} \times 0.635 \mathrm{~cm} \times 0.0381 \mathrm{~cm}$ PZT5H wafer at input levels ranging from $600 \mathrm{~V}$ to $2200 \mathrm{~V}$. As with the PZT5A sample, data was collected at $200 \mathrm{mHz}$ to minimize frequency effects.

For this data set, parameters were estimated through a fit to the $2200 \mathrm{~V}$ data and the resulting model was used to predict the hysteretic $E-P$ relation at lower drive levels. Initial values for $\bar{E}_{c}$ and $\eta$ were obtained from the coercive field $E_{c}=0.9 \times 10^{6} \mathrm{~V} / \mathrm{m}$ and reciprocal slope $\frac{d E}{d P}=3.7 \times 10^{8}$, after saturation, for the $2200 \mathrm{~V}$ data. The absolute least squares functional (32) yielded the parameters $P_{R}=0.04 \mathrm{C} / \mathrm{m}^{2}, \bar{E}_{c}=0.747690 \times 10^{6} \mathrm{~V} / \mathrm{m}, \eta=6.5 \times 10^{8}, c=0.2612 \mathrm{~V}^{2} / \mathrm{m}^{2}, b=2.8425 \times$ $10^{11} \mathrm{~V}^{2} / \mathrm{m}^{2}, C=1.1526 \times 10^{-11}$ and fits depicted in Figure 12. The Euclidean metric (34) yielded the parameter values $P_{R}=0.04 \mathrm{C} / \mathrm{m}^{2}, \bar{E}_{c}=0.698990 \times 10^{6} \mathrm{~V} / \mathrm{m}, \eta=6.5 \times 10^{8}, c=0.3439$ $\mathrm{V}^{2} / \mathrm{m}^{2}, b=3.2407 \times 10^{11} \mathrm{~V}^{2} / \mathrm{m}^{2}, C=8.0932 \times 10^{-12}$ and fits depicted in Figure 13. It is observed that because the absolute metric heavily penalizes discrepancies in high gradient regions, the fits in Figure 12 are very accurate in the coercive region but saturate too quickly. The use of the Euclidean metric provides more uniform penalties throughout the drive range and hence provides a model which is accurate both in switching and saturation.

\section{PZT4}

The final compound that we consider is the hard material PZT4. Data collected from a $3.81 \mathrm{~cm}$ $\times 0.635 \mathrm{~cm} \times 0.381 \mathrm{~cm}$ wafer at input levels of $600 \mathrm{~V}$ through $1800 \mathrm{~V}$ is plotted in Figure 14. For the $1800 \mathrm{~V}$ input, the coercive field $1.42 \times 10^{6} \mathrm{~V} / \mathrm{m}$ reflects that more energy is required to turn dipoles than in the softer PZT5 compounds. 

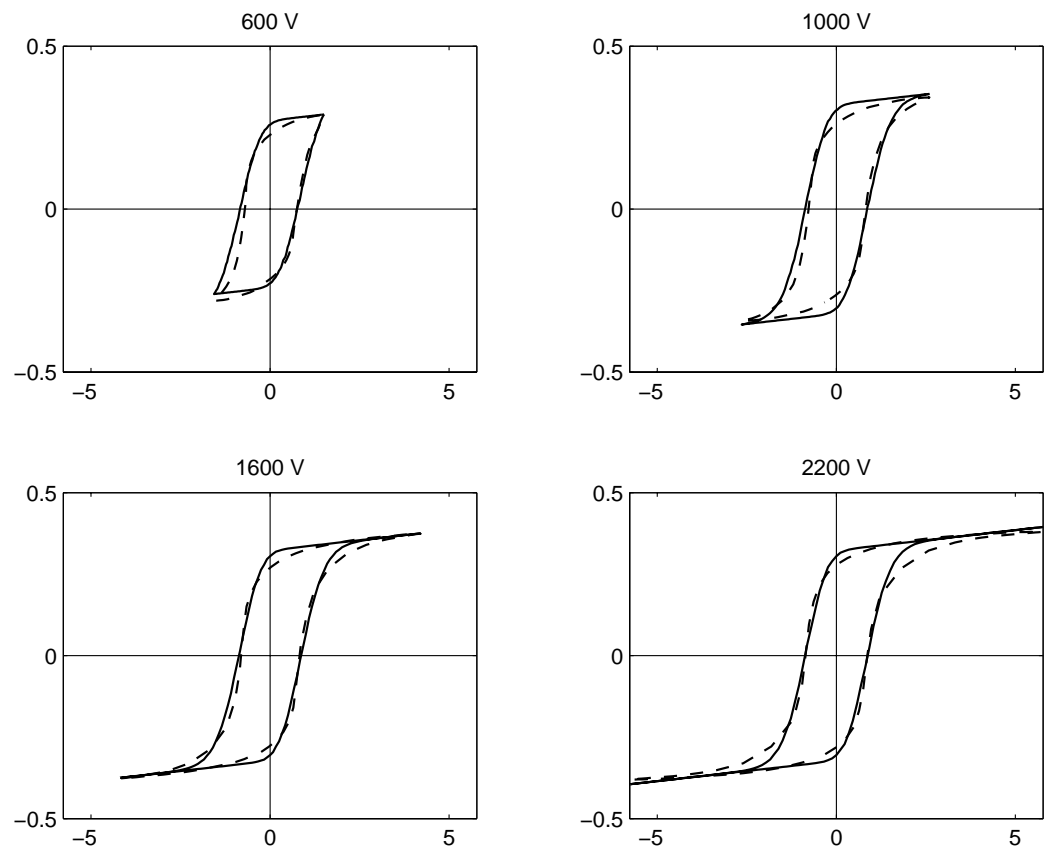

Figure 12. PZT5H data $\left(--_{-}\right)$and model predictions $(-)$with the absolute least squares functional (32) used to determine the parameters $P_{R}=0.04 \mathrm{C} / \mathrm{m}^{2}, \bar{E}_{c}=0.747690 \times 10^{6} \mathrm{~V} / \mathrm{m}$, $\eta=6.5 \times 10^{8}, c=0.2612 \mathrm{~V}^{2} / \mathrm{m}^{2}, b=2.8425 \times 10^{11} \mathrm{~V}^{2} / \mathrm{m}^{2}, C=1.1526 \times 10^{-12}$. Abscissas: electric field $(\mathrm{MV} / \mathrm{m})$, ordinates: polarization $\left(\mathrm{C} / \mathrm{m}^{2}\right)$.
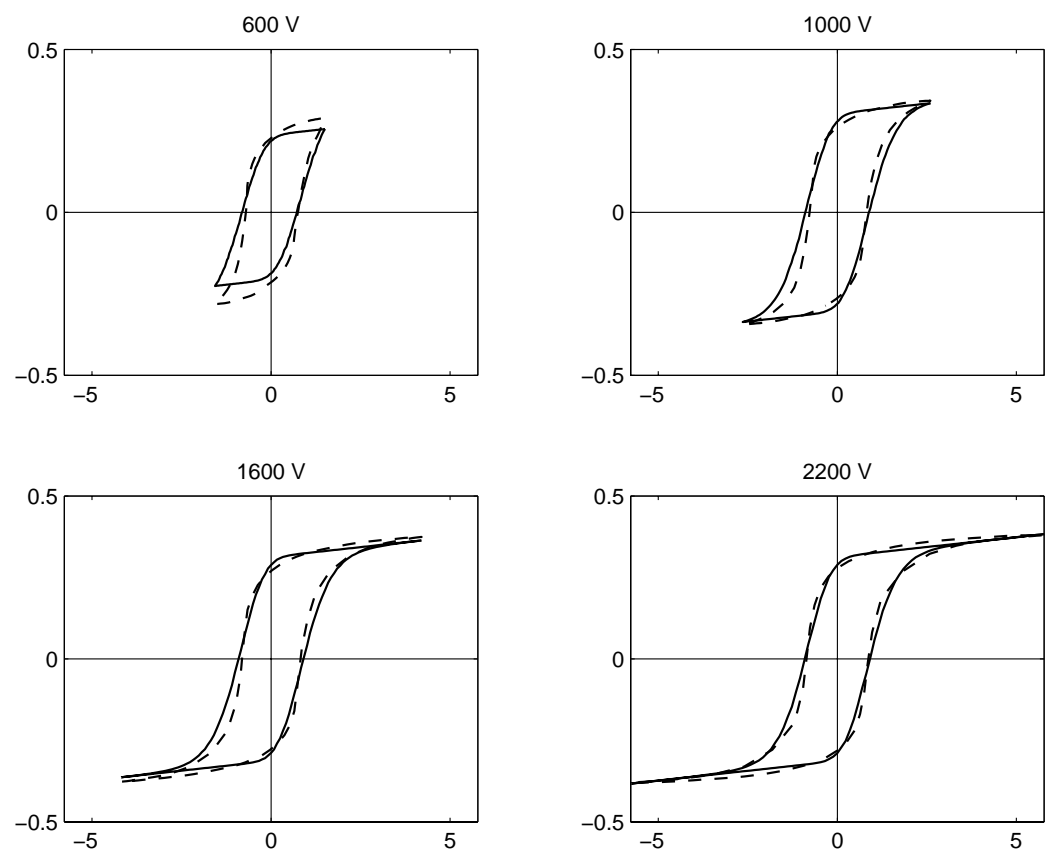

Figure 13. PZT5H data $(---)$ and model predictions $(-)$ with the Euclidean least squares functional (34) used to determine the parameters $P_{R}=0.04 \mathrm{C} / \mathrm{m}^{2}, \bar{E}_{c}=0.698990 \times 10^{6} \mathrm{~V} / \mathrm{m}$, $\eta=6.5 \times 10^{8}, c=0.3439 \mathrm{~V}^{2} / \mathrm{m}^{2}, b=3.2407 \times 10^{11} \mathrm{~V}^{2} / \mathrm{m}^{2}, C=8.0932 \times 10^{-12}$. Abscissas: electric field $(\mathrm{MV} / \mathrm{m})$, ordinates: polarization $\left(\mathrm{C} / \mathrm{m}^{2}\right)$. 

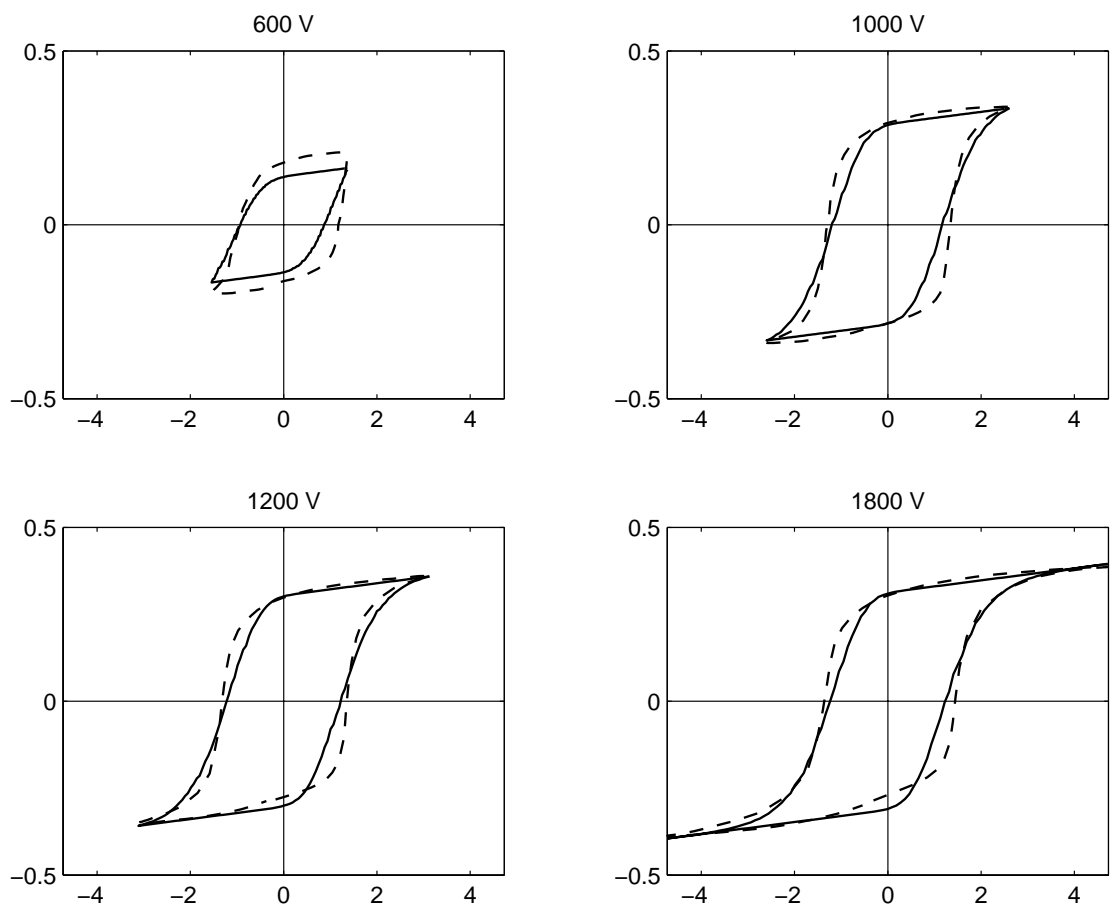

Figure 14. PZT4 data (-- ) and model predictions (-) with the parameters $P_{R}=0.045 \mathrm{C} / \mathrm{m}^{2}$, $\bar{E}_{c}=1.05 \times 10^{5} \mathrm{~V} / \mathrm{m}, \eta=4.0 \times 10^{8}, c=0.3018 \mathrm{~V}^{2} / \mathrm{m}^{2}, b=2.1924 \times 10^{11} \mathrm{~V}^{2} / \mathrm{m}^{2}, C=6.8287 \times 10^{-12}$. Abscissas: electric field $(\mathrm{MV} / \mathrm{m})$, ordinates: polarization $\left(\mathrm{C} / \mathrm{m}^{2}\right)$.

The three functionals (32)-(34) yield roughly equivalent parameters and the model response with the parameters $P_{R}=0.045 \mathrm{C} / \mathrm{m}^{2}, \bar{E}_{c}=1.05 \times 10^{5} \mathrm{~V} / \mathrm{m}, \eta=4.0 \times 10^{8}, c=0.3018 \mathrm{~V}^{2} / \mathrm{m}^{2}$, $b=2.1924 \times 10^{11} \mathrm{~V}^{2} / \mathrm{m}^{2}, C=6.8287 \times 10^{-12}$, estimated through a fit to the $1800 \mathrm{~V}$ data, is compared with the data in Figure 14. It is observed that while model is very accurate in the high drive regime, it does not fully quantify the sharp transition prior to coercivity due to limitations resulting from the choice of the lognormal and normal functions used to respectively characterize the densities of the coercive and effective fields. This tendency is accentuated when the model is subsequently used to predict the $600 \mathrm{~V} E-P$ relation. While the model provides sufficient accuracy for applications such as control design, the discrepancy illustrates that improvements can be made in the model when modeling hard compounds. We are presently investigating the development of higher-order kernels and techniques for identifying general densities to provide additional accuracy for high performance applications which utilize PZT4 transducers.

\subsection{Biased Asymmetric Minor loops}

To illustrate the capability of the model to quantify biased minor loops, the field plotted in Figure 15a was input to the model to generate the E-P relation depicted in Figure 15b. The parameters were taken to be $P_{R}=0.04 \mathrm{C} / \mathrm{m}^{2}, \bar{E}_{c}=0.698990 \times 10^{6} \mathrm{~V} / \mathrm{m}, \eta=6.5 \times 10^{8}, c=$ $0.6877 \mathrm{~V}^{2} / \mathrm{m}^{2}, b=3.2407 \times 10^{11} \mathrm{~V}^{2} / \mathrm{m}^{2}, C=8.0932 \times 10^{-12}$ which are close to those identified for PZT5H (the parameters were modified slightly to highlight aspects of the biased loops). Loop 1 illustrates the capability of the model to characterize the quadratic Raleigh behavior experimentally observed for low drive levels whereas Loop 2 illustrates that the model enforces closure of biased, asymmetric minor loops on the initial polarization curve. The continuity in slope of the initial curve, following the closure of Loop 2, illustrates that the model incorporates the deletion property 


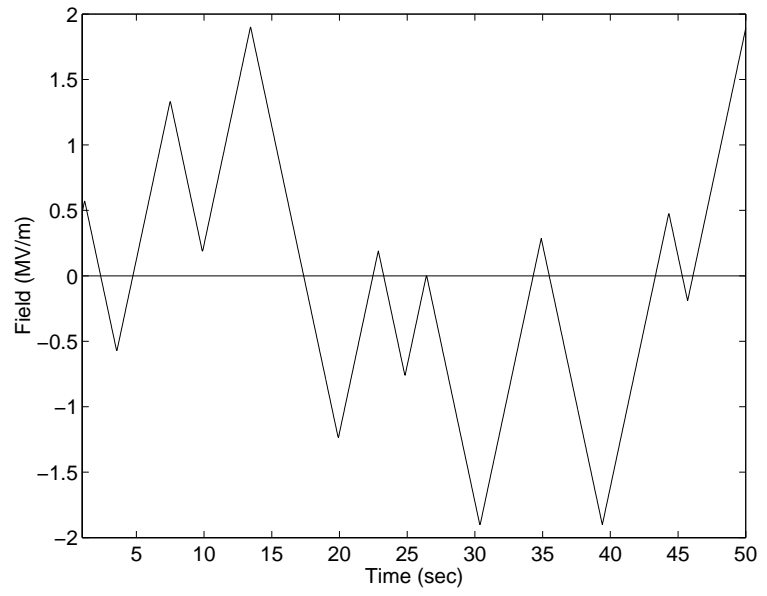

(a)

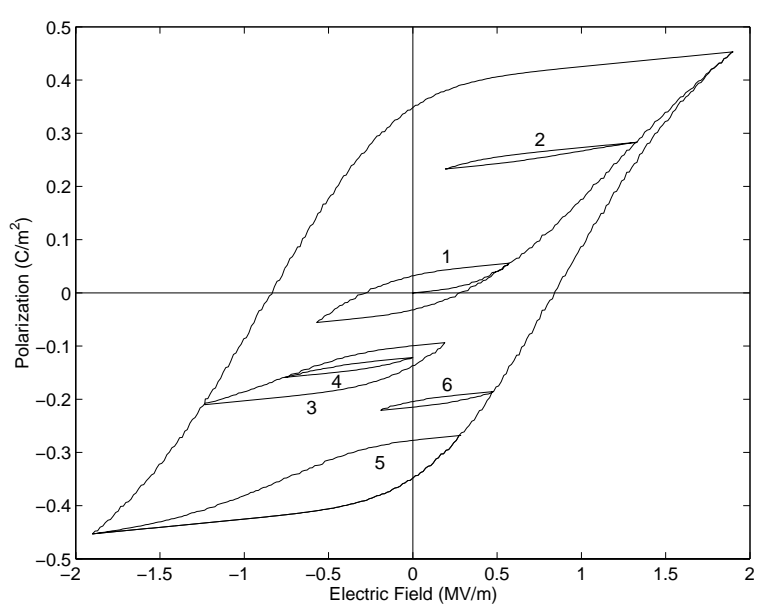

(b)

Figure 15. (a) Field input $E$, and (b) polarization predicted by the discretized model (30).

which, along with congruency, forms one of the necessary and sufficient conditions for classical Preisach models [8]. Loops 3 and 4 illustrate the ability of the model to enforce closure of multiply nested loops while Loop 5 encapsulates biased behavior near saturation. When combined with the experimental results in Section 4.2, the behavior depicted here provides the model with substantial flexibility for material characterization and control design.

\section{Concluding Remarks}

The theory presented in this paper provides a technique for quantifying constitutive nonlinearities and hysteresis inherent to piezoelectric compounds in a manner conducive to bulk material characterization and model-based control design. Through the combination of free energy analysis at the lattice or domain level with the assumption that certain physical parameters are stochastically distributed, macroscopic models having a small number of parameters (5-6) are constructed. Furthermore, several of these parameters can be correlated with physical attributes of the $E$ - $P$ data to facilitate parameter identification and model updating to accommodate changing operating conditions. The model accommodates transient dynamics in the $E-P$ relation, enforces return point memory, and guarantees the closure of both symmetric and biased, asymmetric minor loops. The model does not enforce congruency in the saturation regions of the $E-P$ curve which reflects the measured behavior of the materials in these regions.

The numerical examples and comparison with experimental data illustrate low-frequency, fixedtemperature capabilities of the model. In its present formulation, however, it also incorporates certain relaxation mechanisms and temperature-dependencies. The latter effect has been validated in the context of relaxor ferroelectric compounds (PMN) and a comprehensive set of examples illustrating the quantification of these properties in PZT will appear in a future publication. The model in its present form does not incorporate polarization changes due to variable stresses and these extensions are under current investigation.

The use of Algorithm 1 renders the model highly efficient so that multiloop simulations run in the order of seconds on an aging and overworked workstation. In the algorithm described in [34], the monotonicity of the modeled $E-P$ relation was invoked to construct an inverse model for inclusion in feedforward or feedback loops to linearize (at least approximately) the response of actuators operating 
in nonlinear and hysteretic and nonlinear regimes. The analogous magnetic model and inverse are employed in [17] to construct robust control designs to achieve high accuracy, high speed position control for Terfenol-D transducers. Hence the unified nature of the modeling approach facilitates unified control designs that utilize model-based inverse compensators.

\section{Acknowledgements}

The research of R.C.S. was supported in part through the NSF grant CMS-0099764 and in part by the Air Force Office of Scientific Research under the grant AFOSR-F49620-01-1-0107. The research of S.S. was supported in part by the National Science Foundation through the grant DMI-0134464. The research of J.S. was supported through the NSF grant CMS-0099764 and the NSF subcontract 420-20-78 through Iowa State University. The authors thank Marcelo Dapino, Julie Raye, and an anonymous referee for providing input which helped to improve the paper.

\section{References}

Note: Center for Research in Scientific Computation Technical Reports can be accessed at the web site http://www.ncsu.edu/crsc/reports.html.

[1] J.C. Anderson, Dielectrics, Reinhold Publishing Corporation, New York, 1964.

[2] H.T. Banks, A.J. Kurdila and G. Webb, "Identification of hysteretic control influence operators representing smart actuators Part I: Formulation," Mathematical Problems in Engineering, 3, pp. 287-328, 1997.

[3] H.T. Banks, A.J. Kurdila and G. Webb, "Identification of hysteretic control influence operators representing smart actuators Part II: Convergent approximations," Journal of Intelligent Material Systems and Structures, 8(6), pp. 536-550, 1997.

[4] H.T. Banks, R.C. Smith and Y. Wang, Smart Material Structures: Modeling, Estimation and Control, Masson/John Wiley, Paris/ Chichester, 1996.

[5] W. Chen and C.S. Lynch, "A model for simulating polarization switching and AF-F phase changes in ferroelectric ceramics," Journal of Intelligent Material Systems and Structures, 9, pp. 427-431, 1998.

[6] B.D. Cullity, Introduction to Magnetic Materials, Addison-Wesley, Reading, MA, 1972.

[7] A. Daniele, S. Salapaka, M.V. Salapaka and M. Dahleh, "Piezoelectric scanners for atomic force microscopes: Design of lateral sensors, identification and control," in Proceedings of the American Control Conference, San Diego, CA, pp. 253-257, 1999.

[8] E. Della Torre, Magnetic Hysteresis, IEEE Press, New York, 1999.

[9] M.V. Gandhi and B.S. Thompson, Smart Materials and Structures, Chapman and Hall, New York, 1992.

[10] P. Ge and M. Jouaneh, "Modeling hysteresis in piezoceramic actuators," Precision Engineering, 17, pp. 211-221, 1995. 
[11] D. ter Haar, Elements of Statistical Mechanics, 3rd Ed., Butterworth-Heinemann, Oxford, 1995.

[12] L. Huang and H.F. Tiersten, "An analytic description of slow hysteresis in polarized ferroelectric ceramic actuators," Journal of Intelligent Material Systems and Structures, 9, pp. 417-426, 1998.

[13] S. Lang, Real and Functional Analysis, Springer-Verlag, New York, 1993.

[14] J.E. Miesner and J.P. Teter, "Piezoelectric/magnetostrictive resonant inchworm motor," Proceedings of SPIE, Smart Structures and Materials, Volume 2190, pp. 520-527, 1994.

[15] T. Mitsui, I. Tatsuzaki and E. Nakamura, An Introduction to the Physics of Ferroelectrics, Gordon and Breach Science Publishers, New York, 1976.

[16] A.J. Moulson and J.M. Herbert, Electroceramic: Materials, Properties, Applications, Chapman and Hill, New York, 1990.

[17] J.M. Nealis and R.C. Smith, "Control techniques for high performance nonlinear smart systems," Proceedings of the SPIE, Smart Structures and Materials 2003, to appear.

[18] R.E. Newnham, "Electroceramics," Reports on Progress in Physics, 52, pp. 123-156, 1989.

[19] M. Omura, H. Adachi and Y. Ishibashi, "Simulations of ferroelectric characteristics using a one-dimensional lattice model," Japanese Journal of Applied Physics, 30(9B), pp. 2384-2387, 1991.

[20] M.B. Ozer and T.J. Royston, "Modeling the effect of piezoceramic hysteresis in structural vibration control," Proceedings of SPIE, Smart Structures and Materials, Volume 4326, pp. 89-100, 2001.

[21] N. Papenfuß and S. Seelecke, "Simulation and control of SMA Actuators," SPIE Smart Structures and Materials, Mathematics and Control in Smart Structures, San Diego, CA, pp. 586-595, 1999.

[22] F. Preisach, "Uber die magnetische nachwirkung," Zeitschrift fur Physik, 94, pp. 277-302, 1935.

[23] G. Puglisi and L. Truskinovsky, "Mechanics of a discrete chain with bi-stable elements," Journal of the Mechanics and Physics of Solids, 48, pp. 1-27, 2000.

[24] G. Puglisi and L. Truskinovsky, "Rate independent hysteresis in a bi-stable chain," Journal of the Mechanics and Physics of Solids, 50, pp. 165-187, 2002.

[25] G. Puglisi and L. Truskinovsky, "A mechanism of transformational plasticity," Continuum Mechanics and Thermodynamics, 14(5), pp. 437-457, 2002.

[26] G. Robert, D. Damjanovic and N. Setter, "Preisach modeling of piezoelectric nonlinearity in ferroelectric ceramics," Journal of Applied Physics, 89(9), pp. 5067-5074, 2001.

[27] S. Salapaka, A. Sebastian, J.P. Cleveland and M.V. Salapaka, "High bandwidth nanopositioner: A robust control approach," Review of Scientific Instruments, 73(9), pp. 3232-3241, 2002.

[28] G. Schitter, P. Menold, H.F. Knapp, F. Allgower and A. Stemmer, "High performance feedback for fast scanning atomic force microscopes," Review of Scientific Instruments, 72(8), pp. 3320$3327,2001$. 
[29] S. Seelecke and C. Büskens, "Optimal control of beam structures by shape memory wires," Computer Aided Optimum Design of Structures, V.S. Hernández and C.A. Brebbia (Eds.,) Computational Mechanics Publications, pp. 457-466, 1997.

[30] S. Seelecke and I. Müller, "Shape memory alloy actuators in smart structures - modeling and simulation," Applied Mechanics Reviews, to appear.

[31] R.C. Smith, M.J. Dapino and S. Seelecke, "A free energy model for hysteresis in magnetostrictive transducers," Center for Research in Scientific Computation Technical Report CRSC-TR02-26; Journal of Applied Physics, 93(1), 2003, to appear.

[32] R.C. Smith and C.L. Hom, "Domain wall theory for ferroelectric hysteresis," Journal of Intelligent Material Systems and Structures, 10(3), pp. 195-213, 1999.

[33] R.C. Smith and Z. Ounaies, "A domain wall model for hysteresis in piezoelectric materials," Journal of Intelligent Material Systems and Structures, 11(1), pp. 62-79, 2000.

[34] R.C. Smith, M.V. Salapaka, A. Hatch, J. Smith and T. De, "Model development and inverse compensator design for high speed nanopositioning," Proc. 41st IEEE Conf. Dec. and Control, 2002, Las Vegas, NV, to appear.

[35] R.C. Smith, S. Seelecke, M. Dapino and Z. Ounaies, "A unified model for hysteresis in ferroic materials," Proceedings of the SPIE, Smart Structures and Materials, San Diego, CA, 2003, to appear.

[36] R.C. Smith, S. Seelecke and Z. Ounaies, "A free energy model for piezoceramic materials," Proceedings of the SPIE, Smart Structures and Materials 2002, Volume 4693, pp. 514-524, 2002.

[37] R.C. Smith and S. Seelecke, "An energy formulation for Preisach models," Proceedings of the SPIE, Smart Structures and Materials 2002, Volume 4693, pp. 173-182, 2002.

[38] K. Uchino, Piezoelectric Actuators and Ultrasonic Motors, Kluwer Academic Publishers, Boston, 1997.

[39] X.D. Zhang and C.A. Rogers, "A macroscopic phenomenological formulation of coupled electromechanical effects in piezoelectricity," Journal of Intelligent Material Systems and Structures, 4, pp. 307-316, 1993.

[40] D. Zwillinger, Editor-in-Chief, CRC Standard Mathematical Tables and Formulae, 30th Edition, CRC Press, Boca Raton, 1996. 